\title{
Unravelling the Strategies for Cell wall Biosynthesis used by Salt-Tolerant Proso Millet (Panicum miliaceum L.) under Salt Stress: From Root Structure to Molecular Mechanism
}

\author{
Yuhao Yuan \\ Northwest A\&F University: Northwest Agriculture and Forestry University \\ Chunjuan Liu \\ Northwest A\&F University: Northwest Agriculture and Forestry University \\ Jing Li \\ Northwest A\&F University: Northwest Agriculture and Forestry University \\ Qian Ma \\ Northwest A\&F University: Northwest Agriculture and Forestry University \\ Qinghua Yang \\ Northwest A\&F University: Northwest Agriculture and Forestry University \\ Baili Feng ( $\square$ fengbaili@nwsuaf.edu.cn ) \\ 8213-7478
}

Northwest A\&F University: Northwest Agriculture and Forestry University https://orcid.org/0000-0002-

\section{Research}

Keywords: proso millet roots, salt stress, microstructural, cell wall biosynthesis

Posted Date: February 17th, 2021

DOI: https://doi.org/10.21203/rs.3.rs-221536/v1

License: (c) (i) This work is licensed under a Creative Commons Attribution 4.0 International License. Read Full License 


\section{Abstract}

Background: Considering the twin global problems of increasingly serious energy shortage and effects of salt stress on biofuel plants, breeding of salt-resistant biofuel plant and the discovery of mechanisms for biomass accumulation under salt stress is necessary for energy shortage. Proso millet (Panicum miliaceum L.) is very resilient to abiotic stress, especially to land degradation caused by soil salinization, and its promising as dedicated bioenergy crops for the production of renewable fuels and forage, due to its high photosynthetic efficiency $\mathrm{C}_{4}$ plant and ability to grow in a range of environmental conditions. However, the mechanisms by which the roots of proso millet adapt and tolerate salt-stress are obscure.

Results: In this study, plants of a salt-sensitive cultivar (SS 212) and a salt-tolerant cultivar (ST 47) of proso millet were exposed to severe salt stress and subsequent re-watering. ST 47 exhibited greater salt tolerance and faster recovery than SS 212 , as evidenced by higher increases in total root length (TRL), root surface area (RSA), root tip number (RTN), biomass. Moreover, microstructural analysis showed that relative to SS 212, the roots of ST 47 could maintain more intact internal structures, and thicker cell wall under salt stress, thereby stronger resistance to salt toxicity and maintenance of growth. Digital RNA sequence analysis suggested more genes involved in salt stress resistance were induced in ST 47 than in SS 212. In ST 47 also, re-watering restored most genes that had been induced by salt stress. Results of the Kyoto Encyclopedia of Genes and Genomes (KEGG) pathways analysis revealed that ST 47 maintained better $\mathrm{Na}^{+} / \mathrm{K}^{+}$balance to resist $\mathrm{Na}^{+}$toxicity via a higher capability to restrict $\mathrm{Na}^{+}$uptake, vacuolar $\mathrm{Na}^{+}$sequestration, and $\mathrm{Na}^{+}$exclusion. The mechanism for cell wall biosynthesis in cultivar ST 47 involved the promotion of cell wall composition changes, via efficient regulation of galactose metabolism and biosynthesis of cellulose and phenylpropanoids.

Conclusions: Overall, this study provides valuable salt-resistant biofuel resources and mechanisms for relieving the world energy shortage, which could be applied for the rehabilitation of saline lands.

\section{Background}

Proso millet (Panicum miliaceum L.) is an allotetraploid with two sub-genomes and is one of the earliest domesticated cereal crops around the world [1]. Owing to its close relationship with bioenergy crop switchgrass (Panicum virgatum), and ethanol yield similar to highly fermentable corn (Zea mays) after $72 \mathrm{~h}$ of fermentation, proso millet is also considered as a feedstock for ethanol production [2, 3]. Its originated from Northern China and is now widely grown in the semiarid regions of Asia, Europe, and other continents [4]. Notably, the crop exhibits significantly lower water requirements and higher efficiency of nutrient usage than most other cereal crops [5]. Proso millet is, therefore, the crop of choice for sustainable water use in agricultural production. Besides, proso millet is considered extremely resistant to abiotic stress due to its higher salt and temperature tolerance relative to most cereal crops [6]. Since the release of the proso millet genome $[5,6]$, this species has evidenced an increase in research interest. 
Soil salinization is a significant threat to sustainable agricultural production and ecological balance worldwide [7, 8]. As a major abiotic stress factor, soil salinity severely inhibits plant growth and development [9]. Roots are usually in direct contact with the soil and are, therefore, the first plant organ that gets exposed to high salt concentrations. The subsequent accumulation of excessive $\mathrm{Na}^{+}$affects root architecture via inhibition of root growth and lateral root formation [10]. The root cells, therefore, either have to adapt to the stress or die [11]. Soil salinity could affect the internal root structure in various ways, such as augmenting cell wall lignification, inhibiting cell division, causing disordered root cell arrangement, or inducing apoptosis [12]. Roots form the first line of plant for salt stress, and should, therefore, develop adaptive strategies to resist salt toxicity through root plasticity [13]. However, little research has examined the sophisticated resistance mechanisms that underly plant root response to salt stress $[14,15]$. Therefore, an evaluation of the phenotypic, physiological, microstructural, and gene expression mechanisms by which salt-tolerant crop roots resist salt stress is urgent.

The cell wall is the first barrier that prevents $\mathrm{Na}^{+}$from entering the root cells; thus, cell wall integrity and remodeling plays a crucial role in enhancing plant salt resistance. The excessive $\mathrm{Na}^{+}$in the apoplast of root cells may directly bind to negatively charged cell wall polymers and reduce cell toxicity [16]. On the other hand, excessive $\mathrm{Na}^{+}$replace $\mathrm{Ca}^{2+}$ by directly binding to pectin, which interferes with pectin crosslinking [17]. Lignin is an important cell wall component, and lignified secondary cell walls are involved in plant defense against abiotic stresses, long-distance water transport, and ion uptake [18]. Cell wall thickening by increased deposition of hemicellulose, cellulose, and lignin has been reported to facilitate defense against abiotic stresses [19]. However, it is unclear whether cell wall biosynthesis contributes to the fixation and barrier of $\mathrm{Na}^{+}$.

This present study employed salt sensitive and salt tolerant proso millet cultivars (SS 212 and ST 47, respectively) to investigate the phenotypic, physiological, microstructural, and molecular responses of this crop to salinity. The objective of the present study was to identify key traits and genes that control salinity adaptation in the salt-tolerant cultivar, which could provide salinity related resources and candidate genes.

\section{Methods}

\section{Plant materials and treatments}

This study used seeds of salt sensitive and salt tolerant proso millet cultivars (SS 212 and ST 47, respectively). The seeds were surface disinfected $\left(0.1 \% \mathrm{HgCl}_{2}, 5\right.$ minutes), rinsed (sterile water, three times), sprouted (3-4 days), and cultured in a hydroponics system until the emergence of three leaves (about 10 days). The culture was done in a seedling pot $(12.4 \mathrm{~cm} \times 17.5 \mathrm{~cm})$ under $14 \mathrm{~h}$ light/ $10 \mathrm{~h}$ dark cycles, day/night temperatures of $28 / 18{ }^{\circ} \mathrm{C}$, and relative humidity of $60 \%$. Seedlings were stressed by $1 / 2$ Hoagland's nutrient solution containing $0 \%$ (control) and $1 \% \mathrm{NaCl}$ for 7 days and then transplanted into $1 / 2$

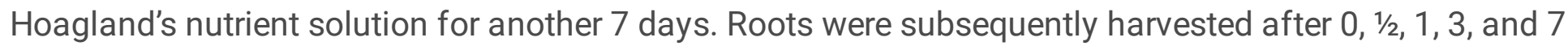


days of salt treatment, and day 7 of subsequent re-watering (RW $7 \mathrm{~d}$ ). The culture solutions were changed every two days throughout the growth period.

\section{Analysis of root system architecture}

Three biological seedling replicates were harvested at $0,1 / 2,1,3$, and 7 days of watering, and RW $7 \mathrm{~d}$, and then divided into aboveground parts and roots. The root systems of the six sample types were scanned with an Epson Perfection V700 Pro scanner (Seiko Epson Corp, Suwa, Japan). Image data were analyzed by the WinRHIZO software (Reagent Instruments Inc., Quebec, Canada) to quantify root parameters, including the total root length (TRL), root surface area (RSA), and root tip number (RTN).

\section{Measurement of biomass and mortality}

To assess effects of salt stress on vegetative growth, the biomass was determined using standard methods recommended by the Organization for Economic Co-operation and Development [20, 21]. The roots and shoots were separated from the seedlings, and dried at $60^{\circ} \mathrm{C}$ to a constant weight in three biological replicates, with 15 seedlings per replicate to determine biomass. Mortality was determined under 7-day salt stress and subsequent re-watering for 7 days. Seedlings were considered to have dead when they heart leaves lose green.

\section{Determination of growth parameter and root physiological parameters}

The levels of root soluble compounds (soluble sugars [SS], soluble proteins [CPr], and proline [PRO]), ascorbate peroxidase (APX), and malondialdehyde (MDA) were determined by the respective Assay Kits (Beijing Solarbio Science \& Technology, Beijing, China), according to the manufacturer's instructions. Root vigor (RV) was determined using a 2,3,5-triphenyl tetrazolium chloride (TTC) assay, as described previously [22].

\section{Determination of $\mathrm{Na}^{+}, \mathrm{K}^{+}, \mathrm{Ca}^{2+}$, and $\mathrm{Mg}^{2+}$ concentrations}

All dry root samples were pulverized using a grinder (A 11 basic Analytical mill, IKA, Staufen, Germany), then $8 \mathrm{ml}$ of nitric acid $\left(\mathrm{HNO}_{3}\right)$ and $2 \mathrm{ml}$ of $30 \%(\mathrm{~m} / \mathrm{m})$ hydrogen peroxide $\left(\mathrm{H}_{2} \mathrm{O}_{2}\right)$ were added to a $0.20 \mathrm{~g}$ sample. Subsequently, the root samples were digested using a microwave digester (Milestone Inc, USA). The contents of $\mathrm{Na}^{+}, \mathrm{K}^{+}, \mathrm{Ca}^{2+}$, and $\mathrm{Mg}^{2+}$ were detected by an atomic absorption spectrophotometer PinAAcle500 (PerkinElmer, Waltham, USA).

\section{Extraction and determination of cell wall composition}


The cell wall was extracted from root, as described previously [23]. The pectin content was determined according to IFJU Method 26. The pectin was coagulated with ethanol in a constant temperature water bath at $85^{\circ} \mathrm{C}$ for $10 \mathrm{~min}$, centrifuged for $15 \mathrm{~min}$ at $4,000 \mathrm{rpm}$, discarded supernatant, and washed with ethanol repeatedly until there was no sugar left. After coagulation and purification, the pectin mixture was added in $0.5 \mathrm{ml} 1 \mathrm{M} \mathrm{NaOH}$, and adjusted to $10 \mathrm{ml}$. After the extracting, the pectin mixture was flocculated with carbazole-ethanol, $\mathrm{H}_{2} \mathrm{SO}_{4}$, measured at $525 \mathrm{~nm}$ using Multiskan $\mathrm{GO}$ (THERMO, Boston, USA), and quantified using a D-Galacturonic acid monohydrate (Sigma-Aldrich) standard curve. The hemicellulose and cellulose contents were determined using the anthrone method $[24,25,26]$. The absorbance of the solution is measured with the Multiskan GO (THERMO, Boston, USA) at $620 \mathrm{~nm}$ and converted to hemicellulose and cellulose contents using a D-Glucose (Sigma-Aldrich) standard curve. The lignin content was determined using acetyl bromide method [27]. The absorbance of the solution is measured with the 1510-04201 Thermo Fisher Microplate Reader (Waltham, USA) at $280 \mathrm{~nm}$.

\section{Microstructural analysis of the root}

Paraffin sections of the root tips were prepared as described previously [28] to examine the root cell structure. Briefly, $3 \mu \mathrm{m}$ thick root transections were cut and taken through the experimental process, mainly including dehydration, dip waxing, embedding, slicing, and safranin 0-fast green staining. Changes in root structure were observed and photographed under epifluorescence microscope (Carl Zeiss, Ober-kochen, Germany). Moreover, root tips were prepared for transmission electron microscope (TEM) using the method described by Meng et al. [29]. Ultrathin sections were stained with uranyl acetate, and lead citrate then examined under a TEM (HT7700, Hitachi, Ltd. Tokyo, Japan).

\section{RNA extraction, cDNA library construction, and digital RNA sequencing}

Total RNA was extracted from the root tissues of three replicates for each treatment time points using an RNA Plant Plus Reagent (Tiangen, Beijing, China), according to the manufacturer's instructions. RNA purity was checked using a NanoDrop 2000 Spectrophotometer (Thermo Scientific, CA, USA), and RNA was resolved on a $1 \%$ agarose gel to check for contamination and degradation. The total RNA integrity was assessed on an Agilent Bioanalyzer 2100 system (Agilent Technologies, CA, USA).

The mRNA was purified from total RNA using poly-T oligo-attached magnetic beads, and then interrupted into short fragments by a fragmentation buffer. Complementary DNA (cDNA) was synthesized using random hexamer primer, M-MuLV Reverse Transcriptase (RNase H), and DNA Polymerase I. The remaining overhangs were then converted into blunt ends via exonuclease/polymerase activities. After adenylation of $3^{\prime}$ ends, NEBNext Adaptors with a hairpin loop structure were ligated to the cDNA to prepare for hybridization and barcoded with a large set of barcode sequences [30]. Then, cDNA fragments (200-250 bp in length) were purified by an AMPure XP system (Beckman Coulter, Beverly, USA), and PCR 
was performed using a Phusion High-Fidelity DNA polymerase, universal PCR primers, and Index $(X)$ Primer. At last, PCR products were purified by the AMPure XP system, and library quality was assessed on the Agilent Bioanalyzer 2100 system (Agilent Technologies, CA, USA).

All the 36 libraries were sequenced on an Illumina Hiseq ${ }^{\text {TM }} 4000$ platform (Illumina, CA, USA) using the PE-150 module, and paired-end $150 \mathrm{bp}$ reads were generated. The adaptor sequences and low-quality reads were filtered by the Trimmomatic tool (v0.33) to clean raw reads [31]. The clean reads obtained after data processing were mapped to the Panicum miliaceum $\mathrm{L}$. genome sequence using TopHat (v2.1.0), and gene expression levels were estimated by fragments per kilobase of transcript per million fragments mapped (FPKM) values.

\section{Analysis of differentially expressed genes (DEGs)}

Differentially expressed genes (DEGs) after $1 / 2,1,3$, and 7 days of salt treatment and RW $7 \mathrm{~d}$ were identified using the DESeq R package (v1.10.1) by comparing the expression levels at these time points with those at $\mathbf{0}$ days. In addition, to evaluate for DEGs among the two cultivars, the gene expression levels of SS 212 were compared with those of ST 47. Genes with a minimal 2-fold changes ( $|\log 2(F C)| \geq 1)$ and a false discovery rate (FDR) of $p \leq 0.05$ were identified as significantly differentially expressed between the two time points [32].

\section{GO and KEGG pathway enrichment analyses}

The GOseq R package (v1.12) and KEGG Orthology Based Annotation System software (KOBAS, v2.0) were used for the analysis of DEGs in the Gene Ontology (GO) and Kyoto Encyclopedia of Genes and Genomes (KEGG) pathways, respectively, and a corrected $P$-value $<0.05$ was chosen as the significance cutoff $[33,34]$.

\section{Validation of RNA-Seq data by RT-qPCR}

We conducted RT-qPCR to validate the RNA-Seq results of 10 randomly selected genes from the roots of both proso millet cultivars. The RNA of three independent biological replicates from each treatment time point was isolated using the RNA Plant Plus Reagent (Tiangen, Beijing, China) according to the manufacturer's protocols. The RNA was reverse transcribed for CDNA synthesis using PrimeScript ${ }^{\mathrm{mm}}$ RT Reagent Kit with gDNA Eraser (Takara Bio, Kusatsu, Japan), following the manufacturers' protocols. RTqPCR was conducted using gene-specific primers designed using the Primer Express (v5.0) software and SYBR® Premix Ex Taq ${ }^{\mathrm{m}}$ II (Tli RNaseH Plus) according to the manufacturer's protocols. Relative gene expression was calculated by the comparative $\mathrm{CT}\left(2^{-\Delta \Delta C T}\right)$ method using the Actin gene as a reference.

\section{Data statistics and analysis}


All results were reported as means \pm standard error, and experiments were performed in three biological replicates. The data were analyzed by the SPSS software version 23.0 (Inc., Chicago, III., USA). Duncan's multiple comparison test was used at the $P<0.05$ level to evaluate significant differences among treatment means.

\section{Results}

\section{Root growth differences in response to salt stress and re- watering in proso millet}

Root growth in the two proso millet cultivars was severely inhibited under salt stress (Fig. 1). Compared with the roots of the control treatment, 7-day salt stress reduced the aboveground, underground, and total biomass of SS 212 by $62.10 \%, 80.00 \%$, and $62.01 \%$, respectively, and by $76.23 \%, 69.65 \%$, and $75.70 \%$, respectively in ST 47. However, subsequent 7-day re-watering increased the aboveground, underground, and total biomass of ST 47 by 2.45 -fold, 2.33 -fold, and 2.44 -fold, but only by 1.33 -fold, 1.29 -fold, and 1.32-fold in SS 212 (Fig. 1B, C, D). Moreover, increases in the TRL, RSA, and RTN of ST 47 (2.62-, 2.40-, and 1.61-folds) were more than that of SS 212 (1.29-, 1.27-, and 1.46-fold) after 7-day salt stress. With subsequent re-watering for seven days, the TRL, RSA, and RTN of ST 47 increased by $82.08 \mathrm{~cm}, 8.01 \mathrm{~cm}^{2}$, and 287.34, which are approximately 5.0-fold higher than that in SS 212 (Fig. 1E-G). These results suggest that ST 47 exhibited higher salt-tolerance and faster recovery than SS 212.

Root physiological differences in response to salt stress and re-watering in both proso millet cultivars

The levels of SS, Cpr, and PRO in the roots of both cultivars were quantified to examine the content of soluble substances under salt stress and re-watering. The SS contents of SS 212 increased 1.56-folds, and by 1.47-fold in ST 47 at $1 / 2$ day; however, ST 47 showed faster recovery than SS 212 upon re-watering (Fig. 2A). The soluble protein contents in ST 47 were more than that in SS 212 during the entire test period (Fig. 2B), indicating that high salt stress inhibits protein synthesis in salt-sensitive cultivars. Salt treatment for $1 / 2$ day increased the PRO contents of ST 47 by 5.87 - fold, whereas 7.29 -fold increase was detected in SS 212 (Fig. 2C).

Besides, we also quantified the MDA, APX, and RV levels of the roots to examine the root structural damage of both proso millet cultivars (Fig. 2). The APX content of ST 47 did not change significantly during the first three days of salt stress, while the APX levels of SS 212 continued to increase significantly with the extension of stress time (Fig. 2D). After salt treatment, substantial increases were detected in the MDA contents of the two cultivars, but the increment and contents in ST 47 were significantly lower than that in SS 212 (Fig. 2E). Besides, the RV contents in ST 47 were considerably higher than that of SS 212 (Fig. 2F).

Cultivar differences in the levels of root $\mathrm{Na}^{+}, \mathrm{K}^{+}, \mathrm{Ca}^{2+}$, and $\mathrm{Mg}^{2+}$ concentrations 
The average concentrations of root $\mathrm{Na}^{+}, \mathrm{K}^{+}, \mathrm{Ca}^{2+}$, and $\mathrm{Mg}^{2+}$ concentrations in both cultivars were similar under control conditions (Fig. 3). At the start of the salt stress, the $\mathrm{Na}^{+}$concentration of both cultivars showed a rapid initial increase and then gradually increased. The $\mathrm{Na}^{+}$concentrations of SS 212 (338.18 $\mathrm{mg} / \mathrm{kg} \mathrm{DW}$ ) at $1 / 2$ day were approximately 1.52 -fold higher than that of ST $47(222.35 \mathrm{mg} / \mathrm{kg} \mathrm{DW})$ (Fig. 3A). Meanwhile, the $\mathrm{K}^{+}$concentration in SS 212 and ST 47 progressively reduced to $11.19 \mathrm{mg} / \mathrm{kg} \mathrm{DW}$ and $18.03 \mathrm{mg} / \mathrm{kg}$ DW at $7 \mathrm{~d}$, respectively, with significant differences between the two cultivars (Fig. 3B). After 7-day salt stress, the $\mathrm{Na}^{+}$concentration in SS 212 and ST 47 decreased significantly but did not return to normal levels ( 136.84 and $133.43 \mathrm{mg} / \mathrm{g} \mathrm{DW}$, respectively) with re-watering (Fig. 3A). In addition, the $\mathrm{K}^{+} / \mathrm{Na}^{+}$of $1 / 2,1,3$, and 7 days salt treatment were found to be $0.082 \pm 0.001,0.051 \pm 0.002,0.044 \pm$ 0.002 , and $0.030 \pm 0.002$ in SS 212 , while the $\mathrm{K}^{+} / \mathrm{Na}^{+}$of ST 47 were found to be $0.095 \pm 0.003,0.091 \pm$ $0.003,0.061 \pm 0.004$, and $0.044 \pm 0.003$ under $1 / 2,1,3$, and 7 days salt treatment (Table S1). The $\mathrm{K}^{+}, \mathrm{Ca}^{2+}$, and $\mathrm{Mg}^{2+}$ concentrations in both cultivars showed a significant decline, but increased significantly with re-watering (Fig. 3B, C, D), especially in ST 47. These results suggest that the uptake and accumulation of $\mathrm{Na}^{+}$had a significant impact on the uptake of $\mathrm{K}^{+}, \mathrm{Ca}^{2+}$, and $\mathrm{Mg}^{2+}$.

Cell wall fractions differences in root responses to salt stress and re-watering

To investigate the impact of salt stress on cell wall, the pectin, cellulose, hemicellulose, and lignin content were determined. The contents of the different cell wall components suggested that there were lower content of pectin in the root cell walls under the salt treatment, compared with control. 7-day salt stress reduced the pectin of SS 212 by $17.33 \%$. However, salt stress had no obvious effects on pectin content of ST 47 in the root cell walls (Fig. 3E). Salt stress also resulted in lower cellulose and hemicellulose contents in the both proso millet roots. 7-day salt stress reduced cellulose and hemicellulose contents of SS 212 by $20.03 \%$ and $11.24 \%$, respectively, and by $17.86 \%$ and $12.69 \%$, respectively in ST 47 (Fig. 3F-G). Moreover, increases in the lignin content of ST 47 (1.16-fold) were more than that of SS 212 (0.99-fold) after 7-day salt stress (Fig. 3H). However, subsequent 7-day re-watering reduced the lignin content of ST 47 by1.11-fold, but increased by 1.02-fold in SS 212 .

Cultivar differences in root microstructural responses to salt stress and re-watering

According to the paraffin section images in Fig. 4, the epidermis of salt-stressed SS 212 revealed significant cell degradation in the root tips, whereas that of ST 47 showed negligible cell disintegration and still maintained standard shape (Fig. 4B, E). The TEM images in Fig. 4G show that under untreated conditions, the root cell structure was complete, with mitochondria distributed around the cell wall in an ellipsoidal shape, clear cristae, even endoplasmic reticulum distribution, and the plasma membrane close to the cell wall. When SS 212 was salt-stressed, the root cell wall became thin due to decomposition, severe plasmolysis occurred, the mitochondrial structure was blurred, and the cristae were disordered. In contrast, the cell wall was thickened in ST 47 roots. Subsequent re-watering did not restore the internal root cell structure of SS 212, but ST 47 regained its normal state. Overall, ST 47 could maintain the root structural integrity to reduce salt toxicity. 
Salt stress and re-watering trigger different transcriptome reprogramming in the two proso millet cultivars

To confirm that the different responses of the two proso millet cultivars to salt stress were due to a significant transcriptomic change, the seedling roots were subjected to salt stress and subsequent recovery. We then performed Illumina HiSeq of 36 RNA samples of the various treatments at different time points (12 samples $\cdot 3$ biological replicates). More than 2.0 billion high-quality reads (average 57 million reads per sample) were mapped to the reference genome. Of these, 24.4 to 58.7 million (78.382.2\%) were mapped on exonic sequences (Table S2). The Cufflinks and Cuffcompare software detected 61,630 transcripts, including 54,901 known and 6,729 novel transcripts (Data S1). Spearman correlation coefficient (SCC) analysis showed that the FPKM values of triplicate samples from each of SS 212 and ST 47 were positively correlated ( $p=0.997$ to 0.998 ) (Fig. S1). About $4.9-5.4 \%$ of genes in both proso millet cultivars was found to be high-expressing (FPKM $\geq 60$ ), and medium-expressing (15मFPKM $\geq 1$ ) and low-expressing (10FPKMX0) (Fig. 5A). Genes with FPKM $\geq 1$ were considered expressed in a given sample [35]. Based on this criterion, a total of 47,648 genes were identified in at least one of the 12 samples. Of these, 40,570 and 42,296 were expressed at individual time points (Data S2; Fig. S2), and 34,614 genes were shared in the two proso millet cultivars (Fig. 5B). Overall, the digital RNA-Seq analyses revealed a substantial reprogramming of the transcriptome of both cultivars in response to salt stress and re-watering.

To validate the digital RNA-Seq data, ten genes were randomly selected from SS 212 and ST 47 (12 samples per cultivar) for RT-qPCR, and primer sequences listed in Table S3. The RT-qPCR analysis results were highly consistent with those of RNA-Seq data (Fig. S3). The correlation coefficient of RT -qPCR and RNA-Seq data for each of the genes was 0.89 , which indicates that the RNA-Seq data were highly accurate.

\section{Transcriptomes Revealed The Relationship Between Salt Stress And Re-watering}

Hierarchical clustering and PCA were performed to verify that the recovery phenotypes induced by salt and subsequent re-watering were associated with transcriptome reprogramming (Fig. 5C, D). As expected, the root transcriptomes of both cultivars showed the highest correlation and clustered together between 0 days and RW $7 d(\rho=0.925$ in SS 212 and $\rho=0.932$ in ST 47, respectively) (Fig. 5C, D). Thus, the transcript patterns between these two time points were more similar in both proso millet cultivars than other time points, especially in ST 47 (Fig. 5C). This result indicates that the salt-induced genes of ST 47 were restored faster than in SS 212 by re-watering.

DEGs reveal differential mechanisms for salt stress response in proso millet

The numbers of DEGs between sample pairs showed significant differential expression between SS 212 and ST 47 overtime under salt stress and re-watering. A total of 3753 and 4600 DEGs were detected in SS 212 and ST 47 at $1 / 2$ day, respectively, as compared with those at day 0, whereas 1745 and 1498 genes 
were differentially expressed after re-watering (RW 7 d), respectively (Fig. 6A, B). A total of 1,881 genes (including 726 transcription factors [TF]-encoding genes) were differentially expressed in ST 47 at $1 / 2$ day as compared with SS 212, while 1,424 genes (including 576 TF-encoding genes) genes were differentially expressed after re-watering (RW $7 \mathrm{~d}$ ) (Fig. S4A). These results strongly suggest that after re-watering, the transcriptome of ST 47 recovers faster and to a greater extent, as compared with SS 212. Therefore, it was once again proven that re-watering restored most salt-induced genes in ST 47. At the same time, these findings are highly consistent with phenotypic, physiological, and microstructural analysis results. Overall, our results demonstrated that ST 47 exhibits a more robust tolerance to salt toxicity, compared to SS 212.

Gene ontology (GO) and KEGG analysis of DEGs were performed to reveal the differences in molecular mechanisms between the two cultivars caused by salt stress and subsequent re-watering. DEGs related to oxidative stress and substance transport were highly expressed in SS 212 (Fig. 6C; Fig. S4B). Meanwhile, DEGs related to stress response, protein modification, and nucleotide-sugar metabolic processes were significantly enriched in ST 47, and it was likely that these $\mathrm{GO}$ terms were involved in resistance to salt toxicity (Fig. 6D; Fig. S4B). Moreover, to understand the difference in molecular mechanism between two cultivars caused by salt stress and subsequent re-watering, the KEGG pathways analysis was performed (Fig. 6E, F; Fig. S4C). One of these pathways that resist and adapt salt toxicity were $\mathrm{Na}^{+}$balance. Another pathway was related to cell wall synthesis (galactose metabolism, cellulose synthesis and phenylpropanoid biosynthesis pathway) (Fig. 7).

Effects of salt stress and re-watering on cell wall synthesis in proso millet

KEGG pathway enrichment analysis indicated that the response of proso millet to salt stress and subsequent re-watering involves a rearrangement of cell wall synthesis. During salt stress, the galactose metabolism pathway genes consisted of higher percentages of up-regulated genes in ST 47 than in SS 212 (Fig. 7). The expression of nine genes that encode four enzymes (alpha-galactosidase, aldose 1epimerase, UDP-sugar pyrophosphorylase, and UTP-glucose-1-phosphate uridylyltransferase) involved in the biosynthesis of UDP-glucose and pectin were up-regulated. Remarkably, the normal expression of alpha-galactosidase in ST 47 was restored after recovery (Fig. 7).

Similarly, 54 genes encoding six enzymes in the cellulose synthesis pathway were identified under salt stress. Interestingly at $1 / 2$ day, eight genes in the cellulose synthesis pathway that encode the six key enzymes (glucosyltransferase, sucrose synthase, cellulose synthase, edo-1,4beta-glucanase, UDP-glucose pyrophosphorylase, hexokinase, and phosphoglucomutase) involved in cellulose microfibril biosynthesis were up-regulated. These results imply that salt stress activated the cellulose synthesis pathway in ST 47 at $1 / 2$ day. After the recovery of SS 212, the expressions of the sucrose synthase (SUS) and hexokinase $(H K)$ were not recovered to the basal level (Fig. 7). Additionally, the critical enzyme cellulose synthase (CesA), which catalyzes the biosynthesis of cellulose from UDP-glucose, was only activated in ST 47 at $1 / 2$ day (Fig. 7). Therefore, these results imply that the up-regulated genes encoding alpha-galactosidase and cellulose synthase may play essential roles in salt resistance via enhancing cell wall biosynthesis. 
We observed that at $1 / 2$ day, seven genes that encode phenylalanine ammonia-lyase (in the phenylpropanoid synthesis pathway) were up-regulated, which are related to guaiacyl and syringyl lignin biosynthesis (Fig. 7). Re-watering up-regulated three genes encodes the key enzymes of the phenylpropanoid synthesis pathway in ST 47. However, the expression of these genes was repressed in SS 212, indicating that re-watering restored growth in salt-stressed plants by enhancing cell wall biosynthesis (Fig. 7).

Expression patterns of key genes involved in $\mathrm{Na}^{+} / \mathrm{K}^{+}$homeostasis under salt stress and re-watering

Sixteen genes related to $\mathrm{Na}^{+}$homeostasis was detected in the roots of both cultivars (Fig. 7). Among them, no expression for non-selective cation channels was detected in the roots of both cultivars under salt stress, while one key gene encoding the HKT1 transporter was more repressed in ST 47 than in SS 212 at $1 / 2$ day (Fig. 7). Four genes encoding AKT1 transporters were repressed to lower levels in ST 47 than in SS 212 under salt stress, while re-watering restored the expressions of AKT1 in SS 212 to higher levels than ST 47. These results further confirm that ST 47 had a higher ability to resist $\mathrm{Na}^{+}$uptake in root cells. Interestingly, salt stress repressed the expression of three genes encoding NHX transporters in SS 212, while those genes maintained high expressions in ST 47. Our results suggest that ST 47 exhibits a higher capacity for vacuolar translocation of $\mathrm{Na}^{+}$. Salt stress also activated one key gene in ST 47 that encodes SOS1 transporters to higher levels than in SS 212 (Fig. 7).

\section{Discussion}

\section{ST 47 roots exhibit stronger resistance and adaptation to salt stress than SS 212}

The phenotype and physiology of plant roots typically undergo a series of adjustments to resist and adapt to high salt environments [36]. These changes mainly involve the root development processes, such as tropism, elongation, and lateral root growth [37, 38]. Compared to SS 212, the underground biomass of ST 47 was less negatively affected by salt stress and demonstrated faster recovery with rewatering (Fig. 1C). The mortality of ST 47 was almost unaffected by salt treatment (Fig. 1E). Moreover, the TRL, RSA, and RTN in ST 47 increased more rapidly than that in SS 212 after re-watering. According to Xu et al. [39], maintenance of a vibrant root system architecture contributes to resistance to salt toxicity. Although the accumulation of soluble substances is effective in resisting salt stress and maintenance of osmotic balance, the synthesis of soluble substances requires a high carbon cost. Therefore, plants could apply this strategy to survive salt stress but grow sub-optimally [40]. Compared to SS 212, the concentrations of SS and PRO in ST 47 increased at a slower rate in response to salt stress (Fig. 2A, C), a phenomenon considered a classic hallmark of halophytes [41]. Since high salt stress inhibits protein synthesis in salt-sensitive crop varieties [42], the soluble protein concentrations of SS 212 in our study remained almost constant under salt stress (Fig. 2B). These results reflect that ST 47 roots resist and adapt to salt toxicity via regulation of root plasticity and physiological mechanisms. 
ST 47 roots exhibit more restoration of salt-induced genes after re-watering than SS 212

Re-watering is an effective strategy for restoring growth in salt-stressed plants [43]. Most previous studies have focused on the physiological responses of plant roots to sodium toxicity. In contrast, only a few have explored the mechanisms for salt tolerance and recovery by determining the effect of re-watering on the restoration of salt-stressed plants. In this study, the root transcriptome of the two cultivars showed the highest correlation before salt stress and after re-watering (Fig. 5C, D). A total of 3,753 and 4,600 DEGs were detected in SS 212 and ST 47 at $1 / 2$ day, respectively (Fig. 8A, C). The DEGs of SS 212 were most enriched in GO terms related to "response to chemical stimulus", "response to oxidative stress", and "drug transport" (Fig. 8B). Meanwhile, the DEGs of ST 47 were enriched in GO terms related to "oxidoreductase activity", "response to chemical stimulus", and "organic substance transport" (Fig. 8D). Re-watering reduced the number of DEGs in the two proso millet cultivars; 1,745 and 1,498 DEGs were detected in SS 212 and ST 47, respectively (Fig. 8E, G). The up-regulated genes in SS 212 were enriched in GO terms related to "quinone and ubiquinone metabolic processes", "response to oxidative stress", and "response to chemical stimulus" (Fig. 8F). In ST 47, the up-regulated genes were enriched in "protein phosphorylation", "protein modification", and "phosphorylation" (Fig. 8H). Time points with high correlation are considered to have similar transcripts [44]. Therefore, it was found that the DEGs restored after re-watering of ST 47 are related to resistance to salt toxicity.

ST 47 roots exhibit higher soluble substances accumulation and better $\mathrm{Na}^{+} / \mathrm{K}^{+}$balance than SS 212

The accumulation of salt (mainly $\mathrm{NaCl}$ ) in the soil to high levels causes osmotic stress and ion toxicity in plants [40,45]. For instance, osmotic stress interferes with the ability of plants to absorb water, thus, plant cells cope via osmotic adjustment, in which soluble compounds are accumulated in cells [46]. This study revealed that the cultivar ST 47 resists the osmotic stress caused by salt damage via synthesizing more soluble substances (SS, CPr, and PRO). The ionic imbalance is associated with a substantial influx of $\mathrm{Na}^{+}[46]$. To resist ion toxicity, root cells in high-salt environments respond by reducing $\mathrm{Na}^{+}$uptake, vacuolar sequestration of $\mathrm{Na}^{+}$, and promoting $\mathrm{Na}^{+}$exclusion to prevent the cytoplasmic accumulation of excess $\mathrm{Na}^{+}$. Firstly, root cells resist salt toxicity by mechanisms that restrict $\mathrm{Na}^{+}$uptake, mainly by downregulating $\mathrm{Na}^{+}$influx at the soil-root boundary (via non-selective cation channels or HKTs) [47]. In this present study, salt stress down-regulated the expressions of genes related to CNGCs and the HKT1 transporter in ST 47, relative to SS 212 (Fig. 7). Secondly, NHX transporters facilitate the vacuolar sequestration of excess cytosolic $\mathrm{Na}^{+}$, which effectively avoids the accumulation of $\mathrm{Na}^{+}$in the cytoplasm [48]. In this study, ST 47 showed more significant up-regulation of genes related to the NHX transporter, compared with SS 212. Finally, the exclusion of excessive $\mathrm{Na}^{+}$in root cells is activated by the SOS signaling pathway [47]. The SOS1 $\mathrm{Na}^{+} / \mathrm{H}^{+}$exchanger is the most prominent component in this pathway, which is required for the extrusion of the excess of $\mathrm{Na}^{+}$out of the cells [49]. Compared with SS 212, the expressions of genes related to the SOS1 transporter were up-regulated in ST 47. Thus, ST 47 showed a lower capacity of restricting the absorption of $\mathrm{Na}^{+}$, higher translocation of $\mathrm{Na}^{+}$to the vacuole, and $\mathrm{Na}^{+}$ exclusion. Moreover, the excessive $\mathrm{Na}^{+}$replaces $\mathrm{K}^{+}$, thus affecting cytosolic enzyme activity and 
metabolism, and constraining plant metabolic processes. Therefore, the maintenance of appropriate $\mathrm{K}^{+}$/ $\mathrm{Na}^{+}$balance is essential for sustained plant growth under salt stress [50]. This study showed that decreasing the $\mathrm{Na}^{+}$and increasing $\mathrm{K}^{+}$concentration in ST 47 was favorable for optimal $\mathrm{K}^{+} / \mathrm{Na}^{+}$ratio under salt stress (Fig. 3A; Table S1), which is essential for resistance to salt toxicity [51]. These results suggest that ST 47 showed a higher ability to discharge $\mathrm{Na}^{+}$from root cells (Fig. 7). Therefore, the results of the present study confirmed that ST 47 has a higher capacity to resist salt toxicity than SS 212 via $\mathrm{Na}^{+}$ balance.

ST 47 shows stronger resistance to salt toxicity via enhancing cell wall biosynthesis

Maintaining cell wall integrity is crucial for plant growth and salt tolerance [52]. Since $\mathrm{Ca}^{2+}$ is involved in secondary cell wall biosynthesis [13], $\mathrm{Na}^{+}$accumulated in the soil-root boundary replaces $\mathrm{Ca}^{2+}$ and directly binds to the root cell wall, thus changing the cell wall components and their chemical properties [53]. In this study, the concentration of $\mathrm{Ca}^{2+}$ in both proso millet cultivars declined significantly following exposure to salt stress ( 0.51 -fold; 0.66 -fold; Fig. 3D). Furthermore, salt toxicity severely destroyed the surface structures, cell shapes, and internal cell structure of SS 212 roots, while ST 47 maintained the root structural integrity to resist salt toxicity. The biosynthesis and deposition of lignin are induced during plant acclimation to salt stress [54]. The lignin content of ST 47 in secondary cell walls was higher response to salt stress (Fig. 3H), the higher lignin content in secondary cell walls acts as barrier to limit $\mathrm{Na}^{+}$entry into root cell, reduce $\mathrm{Na}^{+}$toxicity to plants [55]. Salt stress also promoted reductions in cellulose, concomitantly with increases in lignin content [54]. In this study, the cellulose content of both proso millet cultivars declined significantly following exposure to salt stress (0.80-fold; 0.82 -fold; Fig. 3F). Thus, the regulation of cellulose and lignin deposition in response to salinity may represent an adaptation to resist $\mathrm{Na}^{+}$toxicity [55]. Finally, lignin biosynthetic genes are induced during plant acclimation to salt stress [56]. In this study, genes involved in cell wall biosynthesis were activated and up-regulated in ST 47 under salt stress, including guaiacyl and syringyl lignin biosynthesis, galactose metabolism, and cellulose biosynthesis. Therefore, the transcriptional changes of genes or pathways that participate in cell wall biosynthesis is an essential molecular mechanism leading to cultivar differences in $\mathrm{Na}^{+}$tolerance and accumulation in proso millet.

\section{Conclusions}

The integrated approach employed in the present study has revealed the primary strategies employed by the salt-tolerant proso millet cultivar to adapt to salt stress: 1) Maintaining a vibrant root system architecture. 2) Activating and up-regulating the biosynthesis of soluble substances. 3) Maintaining a smooth root surface, intact internal structure, and a thickened cell wall are conducive to blocking $\mathrm{Na}^{+}$ entering into the cells. 4) Maintain an optimal $\mathrm{Na}^{+}$balance in the root cell via restricting $\mathrm{Na}^{+}$uptake, vacuolar sequestration of $\mathrm{Na}^{+}$, and $\mathrm{Na}^{+}$exclusion from the cytosol. 5) Enhancing cell wall biosynthesis. Therefore, our results highlight that the salt-tolerant cultivar ST 47 employs a coordinated regulation of 
phenotypic, physiological, microstructural, and molecular mechanisms to adapt and resist salt toxicity. These results could guide novel strategies for improving salt resistance in other biofuel plants.

\section{Abbreviations}

TRL: total root length; RSA: root surface area; RTN: root tip number, APX: ascorbate peroxidase; MDA: malondialdehyde; SS: soluble sugars; CPr: soluble proteins; PRO: proline; RV: Root vigor, TTC: 2,3,5triphenyl tetrazolium chloride; cDNA: Complementary DNA; DEGs: Differentially expressed genes; GO: Gene Ontology; KEGG: Kyoto Encyclopedia of Genes and Genomes; TF: transcription factor.

\section{Declarations}

Acknowledgements

The authors would like to thank the Allwegene Tech. (Beijing, China) for assisting in the sequencing and data analysis.

Authors' contributions

B. L. Feng and Y. H. Yuan conceived the project and designed the experiments. Y. H. Yuan and C. J. Liu performed the experiments. Y. H. Yuan, Q. H. Yang, and J. L analyzed the data and conducted bioinformatics analysis. Y. H. Yuan and Q. Ma wrote the original manuscript. All authors revised and approved the final manuscript.

Funding

This work was supported by the National Key Research and Development Program of China (nos. 2019YED1000700 and 2019YFD1000702) and National Natural Science Foundation of China (no. 31371529).

Availability of data and materials

The RNA-seq data obtained in the present study were deposited in the NCBI Sequence Read Archive (https://www.ncbi.nlm.nih.gov/Traces/study/?acc=PRJNA592319).

Ethics approval and consent to participate

Not applicable.

Consent for publication

Not applicable.

Competing interests 
The authors declare that they have no competing interests.

\section{References}

1. Hunt, H.V., Badakshi, F., Romanova, O., Howe, C.J., Jones, M.K., Heslop-Harrison, J.P., 2014. Reticulate evolution in Panicum (Poaceae): the origin of tetraploid broomcorn millet, $P$. miliaceum. J. Exp. Bot. 65, 3165-3175. https://doi.org/10.1093/jxb/eru161.

2. Lu, F., Lipka, A.E., Glaubitz, J., Elshire, R., Cherney, J.H., Casler, M.D., Buckler, E.S., Costich, D.E., 2013. Switchgrass Genomic Diversity, Ploidy, and Evolution: Novel Insights from a Network-Based SNP Discovery Protocol. Plos. Genet. 9, e1003215. https://doi.org/10.1371/journal.pgen.1003215.

3. Rose, D.J., \& Santra, D.K., 2013. Proso millet (Panicum miliaceum L.) fermentation for fuel ethanol production. Ind. Crop. Prod. 43, 602-605. https://doi.org/10.1016/j.indcrop.2012.08.010.

4. Lu, H.Y., Zhang, H.J., Liu, K.B., Wu, N.Q., Li, Y.M., Zhou, K.S., Ye, M.L., Zhang, T.Y., Zhang, H.J., Yang, X.Y., Shen, L.C., Xu, D.K., Li, Q., 2009. Earliest domestication of common millet (Panicum miliaceum L.) in East Asia extended to 10,000 years ago. P. Natl. Sci. 106, 7367-7372. https://doi.org/10.1073/pnas.0900158106.

5. Shi, J.P., Ma, X.X., Zhang, J.H., Zhou, Y.S., Liu, M.X., Huang, L.L., Su, S.L., Zhang, X.B., Gao, X., Zhan, W., Li, P.H., Wang, L., Lu, P., Zhao, H.M., Song, W.B., Lai, J.S., 2019. Chromosome conformation capture resolved near complete genome assembly of broomcorn millet. Nat. Commun. 10, 464-477. https://doi.org/10.1038/s41467-018-07876-6.

6. Zou, C.S., Li, L.T., Miki, D., Li, D.L., Tang, Q.M., Xiao, L.H., Rajput, S., Deng, P., Peng, L., Jia, W., Huang, R., Zhang, M.L., Sun, Y.D., Hu, J.M., Fu, X., Schnable, P.S., Chang, Y.X., Li, F., Zhang, H., Feng, B.L., Zhu, X.G., Liu, R.Y., Schnable, J.C., Chnable, J.C., Zhu, J.K., Zhang, H., 2019. The genome of broomcorn millet. Nat. Commun. 10, 436. https://doi.org/10.1038/s41467-019-08409-5.

7. Amélie, L., \& Zeeb, B., 2019. Curing the earth: a review of anthropogenic soil salinization and plantbased strategies for sustainable mitigation. Sci. Total. Environ. 698, 134235. https://doi.org/10.1016/j.scitotenv.2019.134235.

8. Litalien, A., \& Zeeb, B.A., 2020. Curing the earth: A review of anthropogenic soil salinization and plantbased strategies for sustainable mitigation. Sci. Total. Environ. 698, 134235.

https://doi.org/10.1016/j.scitotenv.2019.134235.

9. Zhu, Y.F., Jia, X.M., Wu, Y.X., Hu, Y., Cheng, L., Zhao, T., Huang, Z.C., Wang, Y.X., 2020. Quantitative proteomic analysis of Malus halliana exposed to salt-alkali mixed stress reveals alterations in energy metabolism and stress regulation. Plant. Growth. Regul. 90, 205-222. https://doi.org/10.1007/s10725-019-00563-6.

10. Qin, H., Wang, J., Chen, X.B., Wang, F.F., Peng, P., Zhou, Y., Miao, Y.C., Zhang, Y.Q., Gao, Y.D., Qi, Y.D., Zhou, J.H., Huang, R.F., 2019. Rice OsDOF15 contributes to ethylene-inhibited primary root elongation under salt stress. New. Phytol. 223, 798-813. https://doi.org/10.1111/nph.15824. 
11. Sunitha, M., Srinath, T., Reddy, V.D., Rao, K.V., 2016. Pigeonpea hybrid-proline-rich protein (CcHyPRP) confers biotic and abiotic stress tolerance in transgenic rice. Front. Plant. Sci. 6, 1167. https://doi.org/10.3389/fpls.2015.01167.

12. Rossi, L., Zhang, W., Ma, X., 2017. Cerium oxide nanoparticles alter the salt stress tolerance of Brassica napus $L$. by modifying the formation of root apoplastic barriers. Environ. Pollut. 229, 132. https://doi.org/ 10.1016/j.envpol.2017.05.083.

13. Zhu, J.K, 2016. Abiotic stress signaling and responses in plants. Cell. 167, 313-324. https://doi.org/ 10.1016/j.cell.2016.08.029.

14. Shen, Q.F., Yu, J.H., Fu, L.B., Wu, L.Y., Dai, F., Jiang, L.X., Wu, D.Z., Zhang, G.P., 2017. Ionomic, metabolomic and proteomic analyses reveal molecular mechanisms of root adaption to salt stress in Tibetan wild barley. Plant. Physiol. 123, 319-330. https://doi.org/10.1016/j.plaphy.2017.12.032.

15. Sun, X.C., Wang, Y., Xu, L., Li, C., Zhang, W., Luo, X.B., Jiang, H.Y., Liu, L.W., 2017. Unraveling the root proteome changes and its relationship to molecular mechanism underlying salt stress response in radish (Raphanus sativus L.). Front. Plant. Sci. 8, 1192. https://doi.org/10.3389/fpls.2017.01192.

16. Shomer, I., Novacky, A.J., Pike, S.M., Yermiyahu, U., Kinraide, T.B., 2003. Electrical potentials of plant cell walls in response to the ionic environment. Plant. Physiol. 133, 411-422. https://doi.org/10.1104/pp.103.024539.

17. Sánchez-Aguayo, I., Rodrigues-Galán, J.M., Garcia, R., Torreblanca, J., Pardo, J.M., 2004. Salt stress enhances xylem development and expression of Sadenosyl-L-methionine synthase in lignifying tissues of tomato plants. Planta. 220, 278-285. https://doi.org/10.1007/s00425-004-1350-2.

18. Shafi, A., Pal, A.K., Sharma, V., Kalia, S., Kumar, S., Ahuja, P.S., Singh, A.K., 2017. Transgenic potato plants overexpressing SOD and APX exhibit enhanced lignification and starch biosynthesis with improved salt stress tolerance. Plant. Mol. Biol. Rep. 35, 504-518. https://doi.org/10.1007/s11105017-1041-3.

19. OECD., 2003a. OCED guideline for the testing of chemicals, proposal for updating guideline 208, Terrestrial plant test: seedling emergence and seedling growth test. OCED, Paris, France.

20. OECD., 2003b. OECD guideline for the testing of chemicals, proposal for a new guideline 227, Terrestrial plant test: vegetative vigour test. OECD, Paris, France.

21. Lopez Del Egido L., Navarro-Miró, D., Martinez-Heredia, V., Toorop, P.E., lannetta, P.P., 2017. A spectrophotometric assay for robust viability testing of seed batches using 2, 3, 5-Triphenyl Tetrazolium chloride: using Hordeum vulgare L, as a model. Front. Plant. Sci. 8, 747. https://doi.org/10.3389/fpls.2017.00747.

22. Hu, H., Brown, P.H., 1994. Localization of boron in cell walls of squash and tobacco and its association with pectin (Evidence for a structural role of boron in the cell wall). Plant. Physiol. 105, 681-689. https://doi.org/10.1104/pp.105.2.681.

23. Viles, F.J., \& Silverman, L., 1949. Determination of starch and cellulose with anthrone. Anal. Chem. 21, 950-953. https://doi.org/10.1021/ac60032a019. 
24. Wang, L., Jin, P., Wang, J., Jiang, L.L., Shan, T.M., Zheng, Y. H., 2015. Effect of b-aminobutyric acid on cell wall modification and senescence in sweet cherry during storage at $20^{\circ} \mathrm{C}$. Food. Chem. $175,471-$ 477. https://doi.org/10.1016/j.foodchem.2014.12.011.

25. Chen, Y.H., Hung, Y.C., Chen, M.Y., and Lin, H.T. 2017. Effects of acidic electrolyzed oxidizing water on retarding cell wall degradation and delaying softening of blueberries during postharvest storage. LWT Food Sci. Technol. 84, 650-657. https://doi.org/10.1016/j.Iwt.2017.06.011.

26. Hatfield, R.D., \& Fukushima R.S., 2005. Can lignin be accurately measured? Crop. Sci. 45, 832-839. https://doi.org/10.2135/cropsci2004.0238.

27. Qi, F.F., Zha, Z.Y., Du, L., Feng, X.J., Wang, D.N., Zhang, D., Fang, Z.D., Ma, L.J., Jin, Y.D., Xia, C.Q., 2014. Impact of mixed low-molecular-weight organic acids on uranium accumulation and distribution in a variant of mustard (Brassica juncea var. tumida). J. Radioanal. Nucl. Ch. 302, 149-159. https://doi.org/10.1007/s10967-014-3279-7.

28. Meng, J., Xu, T., Wang, Z., Fang, Y., Xi, Z., Zhang, Z., 2014. The ameliorative effects of exogenous melatonin on grape cuttings under water-deficient stress: antioxidant metabolites, leaf anatomy, and chloroplast morphology. J. Pineal. Res. 57, 200-212. https://doi.org/10.1111/jpi.12159.

29. Smith, T., Heger, A., Sudbery, I., 2017. UMI-tools: modeling sequencing errors in Unique Molecular Identifiers to improve quantification accuracy. Genome. Res. 27, 491-499. https://doi.org/10.1101/gr.209601.116.

30. Bolger, A.M., Lohse, M., Usadel, B., 2014. Trimmomatic: a flexible trimmer for Illumina sequence data. Bioinformatics. 30, 2114-2120. https://doi.org/10.1093/bioinformatics/btu170.

31. Storey, J.D., \& Tibshirani, R., 2003. Statistical significance for genomewide studies. P. Natl. Acad. Sci. USA. 100, 9440-9445. https://doi.org/10.1073/pnas.1530509100.

32. Mao, X.Z., Cai, T., Olyarchuk, J.G., Wei, L.P., 2005. Automated genome annotation and pathway identification using the KEGG Orthology (KO) as a controlled vocabulary. Bioinformatics. 21, 37873793. https://doi.org/10.1093/bioinformatics/bti430.

33. Young, M.D., Wakefield, M.J., Smyth, G.K., Oshlack, A., 2010. Gene ontology analysis for RNA-seq: accounting for selection bias. Genome. Biol. 11, 1-12. https://doi.org/10.1186/gb-2010-11-2-r14.

34. Chen, Q., Pei, D., Li, J.Y., Jing, C.N., Man, Y.H., 2017. The antenna transcriptome changes in mosquito Anopheles sinensis, pre- and post-blood meal. PLoS One. 12, e0181399. https://doi.org/10.1371/journal.pone.0181399.

35. Nguyen, L.V., Bertero, D., Nguyen, L.V., 2020. Genetic variation in root development responses to salt stresses of quinoa. J. Agro. Crop. Sci. 206, 538-547. https://doi.org/10.1111/jac.12411.

36. Galvan-Ampudia, C.S., Julkowska, M.M., Darwish, E., Gandullo, J., Korver, R.A., Brunoud, G., Haring, M.A., Munnik, T., Vernoux, T., Testerink, C., 2013. Halotropism is a response of plant roots to avoid a saline environment. Curr. Biol. 23, 2044-2050. https://doi.org/10.1016/j.cub.2013.08.042.

37. Placido, D.F., Sandhu, J., Sato, S.J., Nersesian, N., Quach, T., Clemente, T.E., Staswick, P.E., Walia, H., 2020. The LATERAL ROOT DENSITY gene regulates root growth during water stress in wheat. Plant. Biotechnol. J. 18, 1955-1968. https://doi.org/10.1111/pbi.13355. 
38. Xu, N., Chu, Y.L., Chen, H.L., Li, X.X., Wu, Q., Jin, L., Wang, G.X., Huang, J.L., 2018. Rice transcription factor OsMADS25 modulates root growth and confers salinity tolerance via the ABA-mediated regulatory pathway and ROS scavenging. PLOS. Genetics. 14, e1007662. https://doi.org/10.1371/journal.pgen.1007662.

39. Munns, R., \& Gilliham, M., 2015. Salinity tolerance of crops - what is the cost? New. Phytol. 208, 66673. doi:10.1371/10.1111/nph.13519.

40. Zhao, C., Zhang, H., Song, C., Zhu, J.K., Shabala, S., 2020. Mechanisms of plant responses and adaptation to soil salinity. The. Innovation. 1, 100017. https://doi.org/10.1016/j.xinn.2020.100017.

41. Sun, J.K., He, L., Li, T., 2019. Response of seedling growth and physiology of Sorghum bicolor(L.) Moench to saline-alkali stress. PLoS One. 14, e0220340.

https://doi.org/10.1371/journal.pone.0220340.

42. Yang, L.L., Zhao, Y., Zhang, Q., Cheng, L., Han, M., Ren, Y.Y., Yang, L.M., 2019. Effects of drought-rewatering-drought on the photosynthesis physiology and secondary metabolite production of Bupleurum chinense DC. Plant. Cell. Rep. 38, 1181-1197. https://doi.org/10.1007/s00299-019-024368.

43. Garg, R., Singh, V.K., Rajkumar, M.S., Kumar, V. Jain, M., 2017. Global transcriptome and coexpression network analyses reveal cultivar-specific molecular signatures associated with seed development and seed size/weight determination in chickpea. Plant. J. 91, 1088-1107. https://doi.org/10.1111/tpj.13621.

44. Zelm, E.V., Zhang, Y.X., Testerink, C., 2020. Salt tolerance mechanisms of plants. Annu. Rev. Plant. Biol. 71, 24.1-24.31. https://doi.org/10.1146/annurev-arplant-050718-100005.

45. Assaha, D.V.M., Akihiro, U., Hirofumi, S., Rashid, A.Y., Yaish, M.W., 2017. The role of $\mathrm{Na}^{+}$and $\mathrm{K}^{+}$ transporters in salt stress adaptation in glycophytes. Front. Physiol. 8, 509. https://doi.org/10.3389/fphys.2017.00509.

46. Albaladejo, I., Meco, V., Plasencia, F., Flores, F.B., Bolarin, M.C., Egea, I., 2016. Unravelling the strategies used by the wild tomato species Solanum pennellii to confront salt stress: from leaf anatomical adaptations to molecular responses. Environ. Exp. Bot. 135, 1-12. https://doi.org/10.1016/j.envexpbot.2016.12.003.

47. Li, W.H., Du, J., Feng, H.M., Wu, Q., Xu, G.H, Shabala, S., Yu, L., 2020. Function of NHX-type transporters in improving rice tolerance to aluminum stress and soil acidity. Planta. 251, 71. https://doi.org/10.1007/s00425-020-03361-x.

48. Wang, W.Y., Liu, Y.Q., Duan, H.R., Yin, X.X., Cui, Y.N., Chai, W.W., Song, X., Flowers, T.J., Wang, S.M., 2020. SsHKT1;1 is coordinated with SsSOS1 and SSNHX1 to regulate $\mathrm{Na}^{+}$homeostasis in Suaeda salsa under saline conditions. Plant. Soil. 449, 117-131. https://doi.org/10.1007/s11104-020-04463x.

49. Ma, C., Xin, M., Feldmann, K.A., Wang, X., 2014. Machine learning-based differential network analysis: A study of stress-responsive transcriptomes in Arabidopsis. Plant. Cell. 26, 520-537. https://doi.org/10.1105/tpc.113.121913. 
50. Meng, J., Ma, M., Luo, J.C., Wu, Q., 2019. Salt accumulation and secretion patterns of Glycyrrhiza uralensis in saline habitats. Flora. 259, 151. https://doi.org/10.1016/j.flora.2019.151449.

51. Feng, W., Kita, D., Peaucelle, A., Cartwright, H.N., Doan, V., Duan, Q., Liu, M.C., Maman, J., Steinhorst, L., Schmitzthom, I., Yvon, R., Kudla, J., Wu, H.M., Cheung, A.Y., Dinneny, J.R., 2018. The FERONIA receptor kinase maintains cell-wall integrity during salt stress through $\mathrm{Ca} 2+$ signaling. Curr. Biol. 28, 666-675. https://doi.org/10.1016/j.cub.2018.01.023.

52. Morris, E.R., Powell, D.A., Gidley, M.J., Rees, D.A., 1982. Conformations and interactions of pectins: I. Polymorphism between gel and solid states of calcium polygalacturonate. J. Mol. Bio. 155, 507-516.

53. Oliveira, D.M., Mota, T.R., Salatta, F.V., Sinzker, R.C., Konc ití́ková, R., Kopečný, D., Simister, R., Silva, M., Goeminne, G., Morreel, K., Rencoret, J., Gutiérrez, A., Tryfona, T., Marchiosi, R., Dupree, P., Río, J.C.D., Boerjan, W., McQueen-Mason, S.J., Gomez, L.D., Ferrarese-Filho, O., Santos, W.D.D., 2020. Cell wall remodeling under salt stress: Insights into changes in polysaccharides, feruloylation, lignification, and phenolic metabolism in maize. Plant. Cell. Environ. 43, 2172-2191. https://doi.org/10.1111/pce.13805.

54. Byrt, C.S., Munns, R., Burton, R.A., Gilliham, M., Wege, S., 2018. Root cell wall solutions for crop plants in saline soils. Plant. Sci. 269, 47-55. https://doi.org/10.1016/j.plantsci.2017.12.012.

55. Hu, P., Zhang, K.M., Yang, C.P., 2019. BpNAC012 positively regulates abiotic stress responses and secondary wall biosynthesis. Plant. Physiol. 179, 700-717. https://doi.org/10.1104/pp.18.01167.

Figures 
$\mathbf{A}$

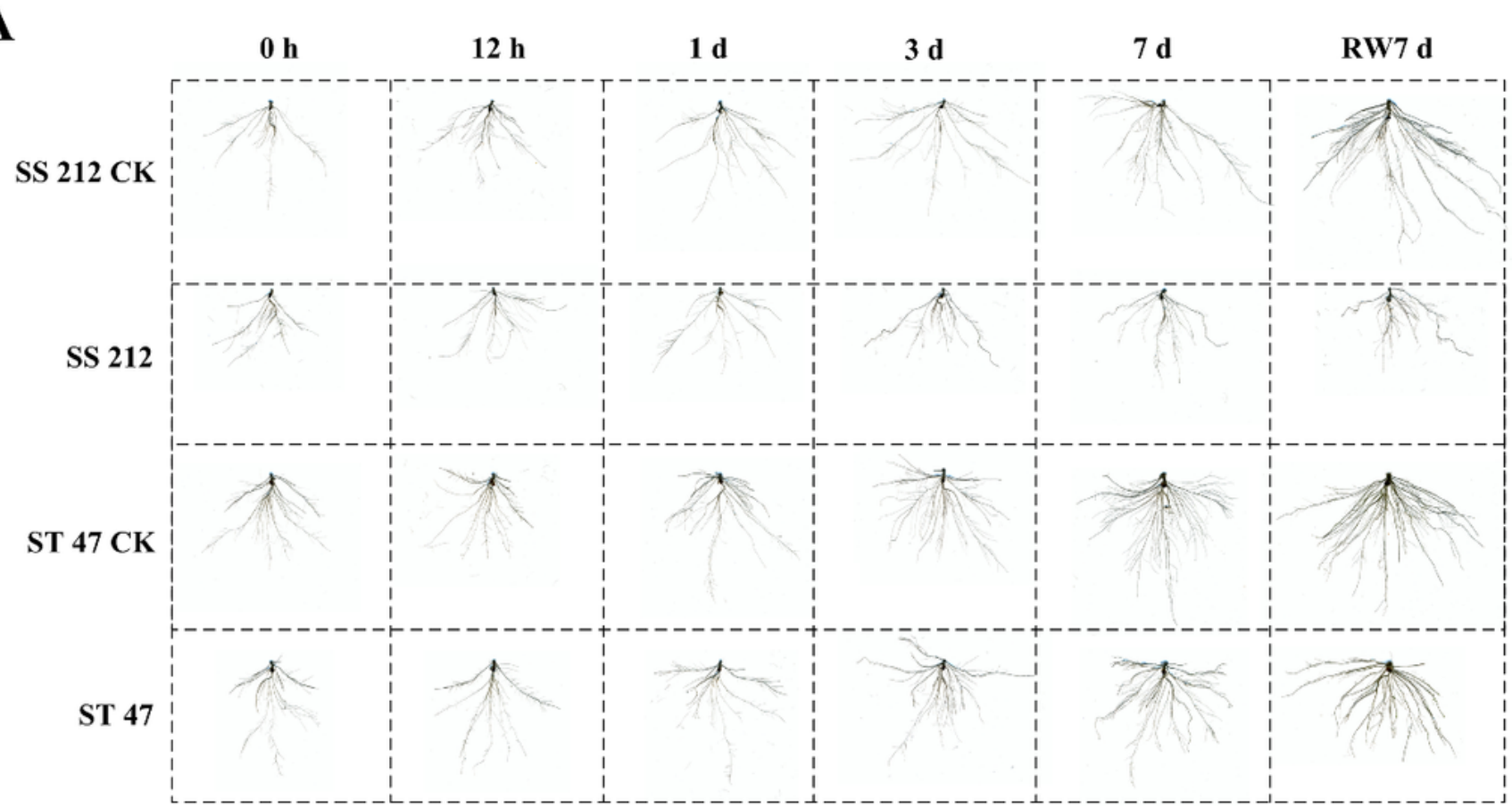

B

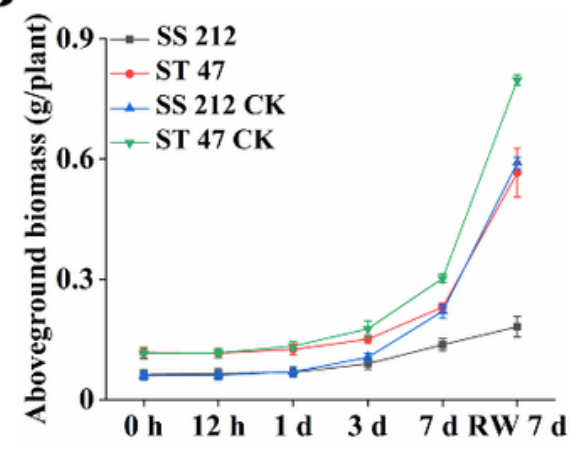

$\mathbf{E}$

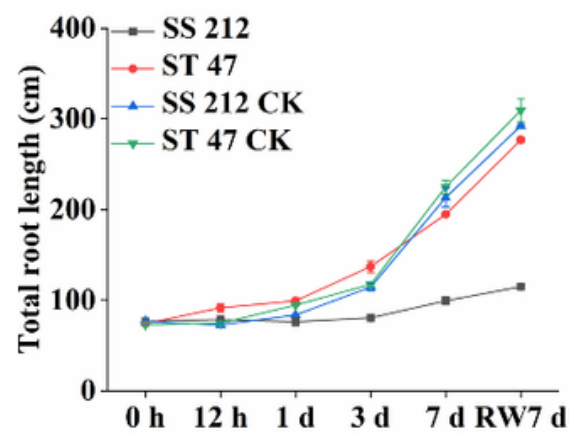

C

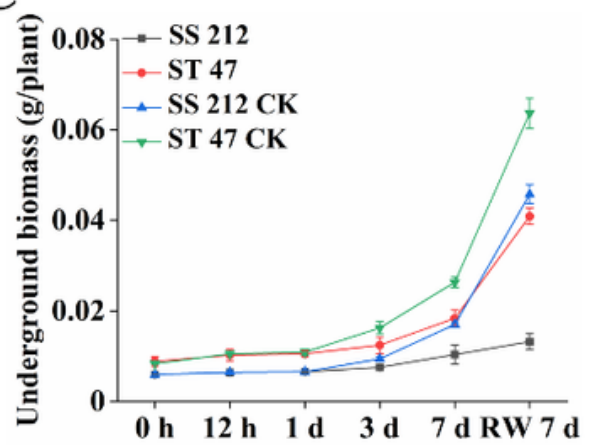

$\mathbf{F}$

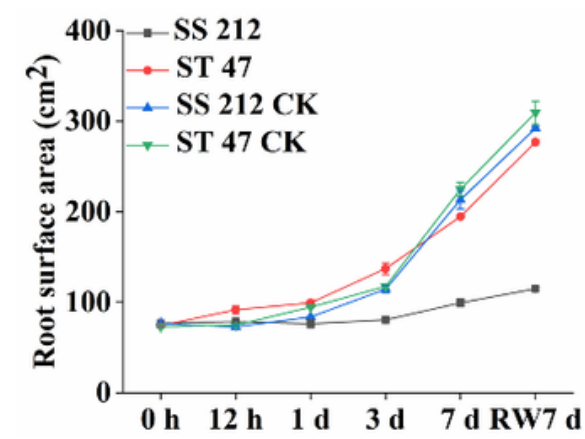

D

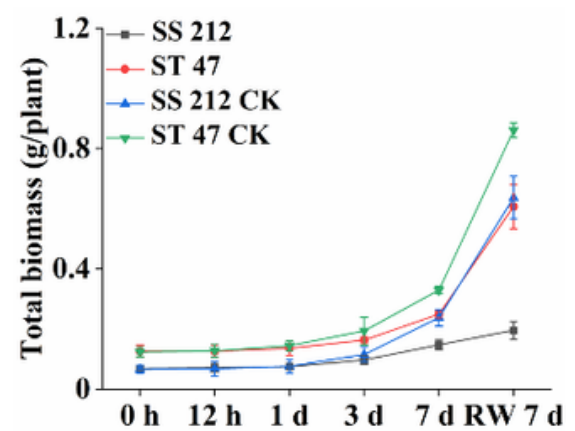

G

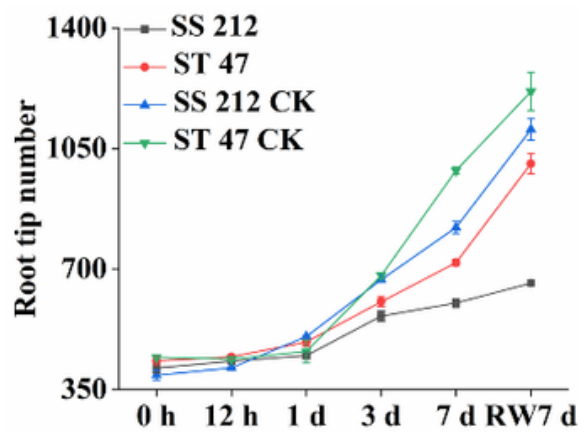

Figure 1

Growth of SS 212 and ST 47 roots with or without salt treatment. (A) Phenotypes under salt treatment. (B) Aboveground biomass. (C) Underground biomass. (D) Total biomass. (E) Total root length. (F) Root surface area. (G) Root tip number. 
A

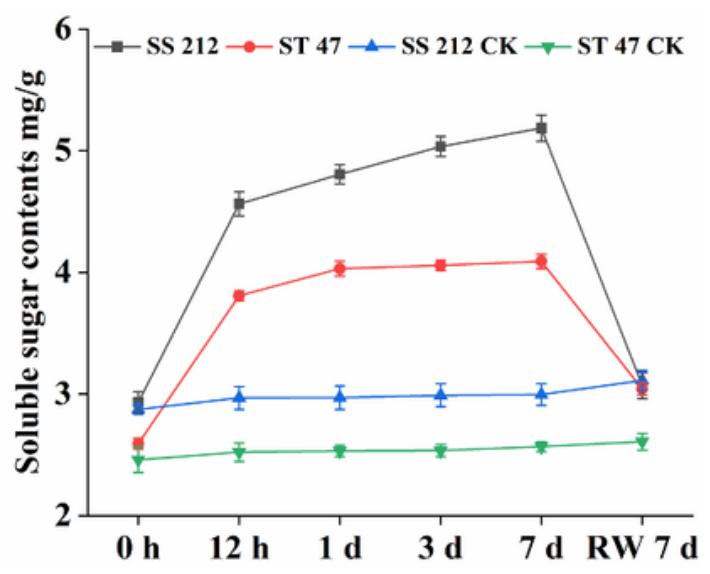

C

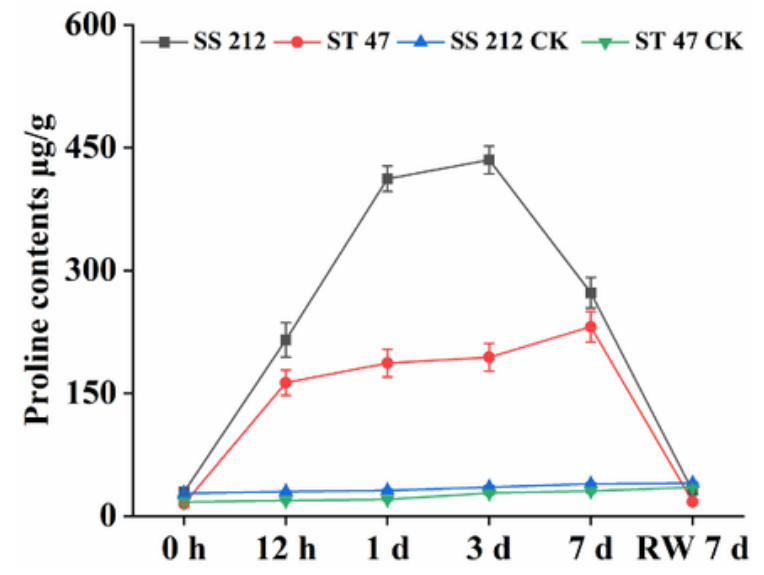

E

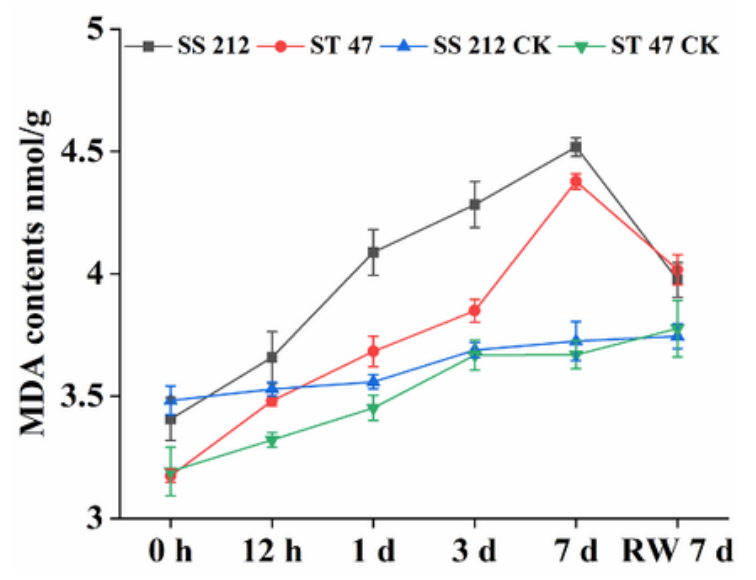

B

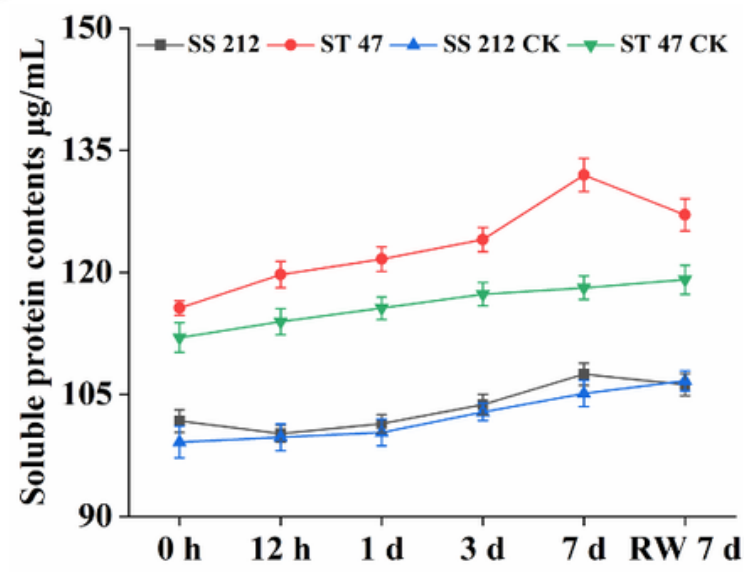

D

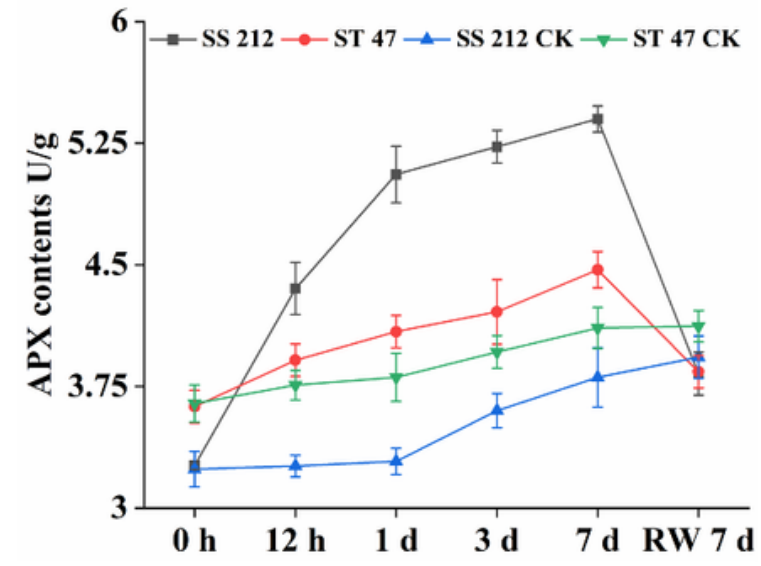

F

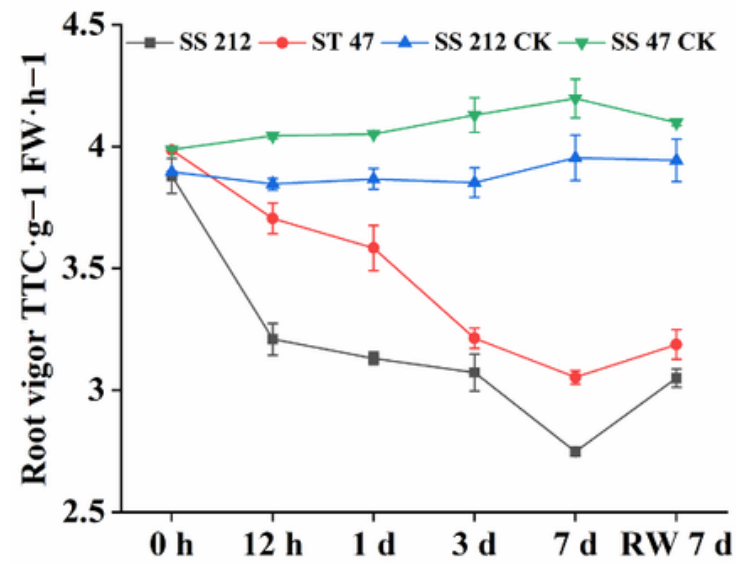

Figure 2

Physiological dynamics between SS 212 and ST 47 in response to salt stress and re-watering. (A) Root soluble sugar concentrations. (B) Root soluble protein concentrations. (C) Root proline concentrations. (D) Root APX contents. (E) Root MDA contents. (F) Root vigor. 
A

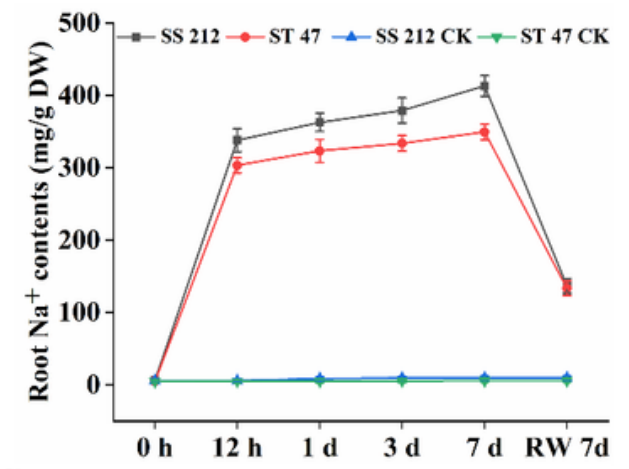

C

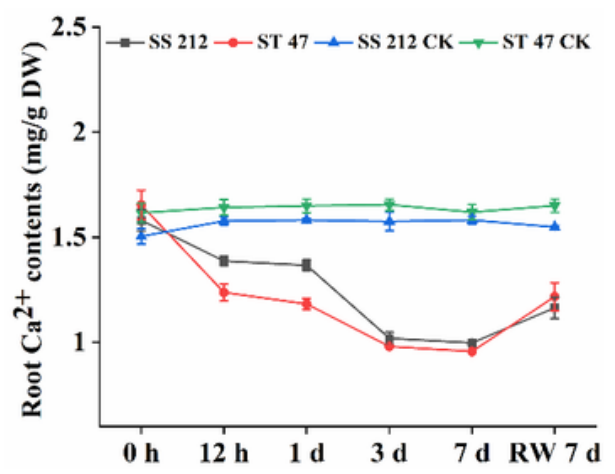

E

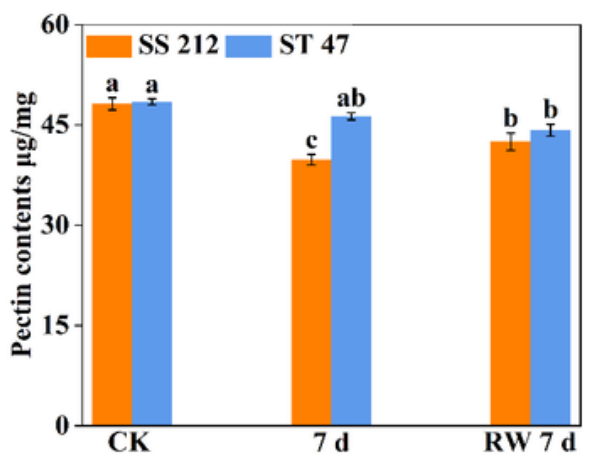

G

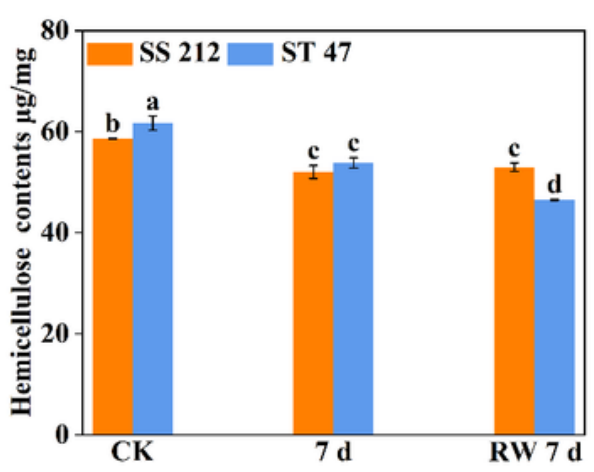

B

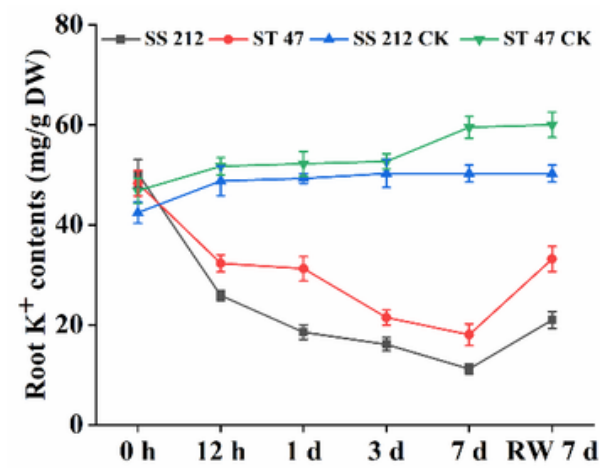

D

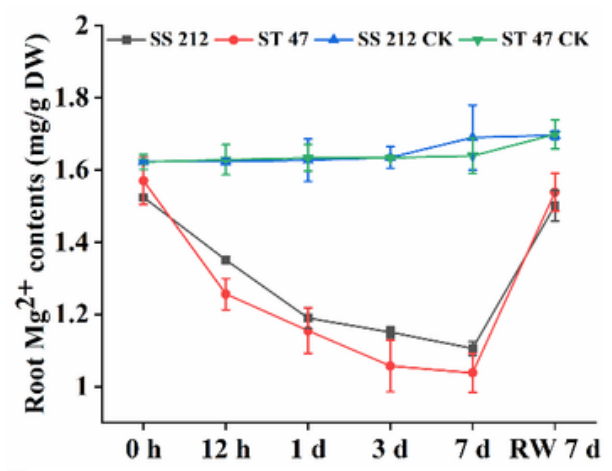

F

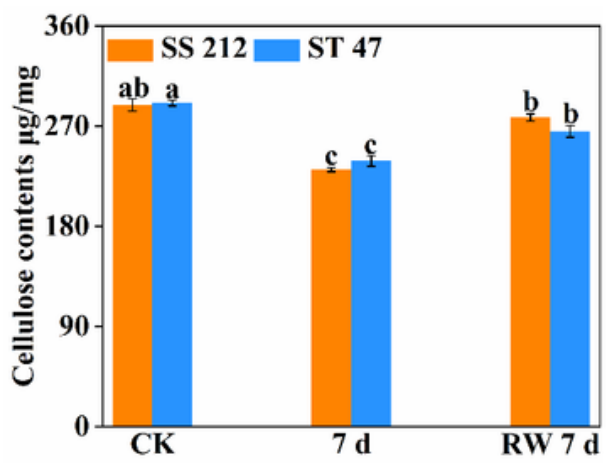

H

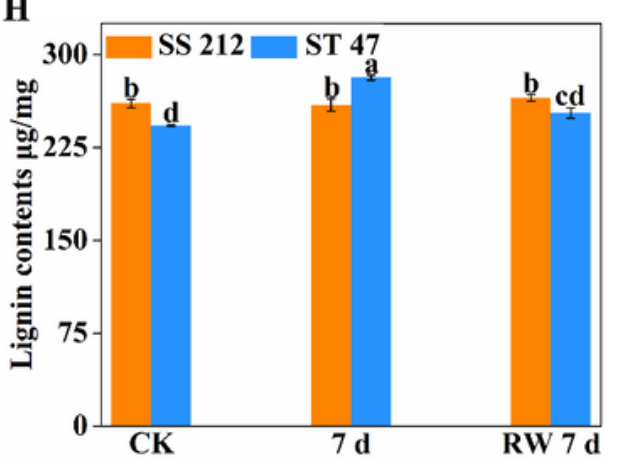

Figure 3

Ion contents and cell wall fractions in the roots of SS 212 and ST 47 under normal conditions, salt stress, and after re-watering. (A) Contents of Na+. (B) Contents of K+. (C) Contents of Ca2+. (D) Contents of Mg2+. (E) Contents of pectin. (F) Contents of cellulose. (G) Contents of hemicellulose. (H) Contents of lignin. 


\section{Control}

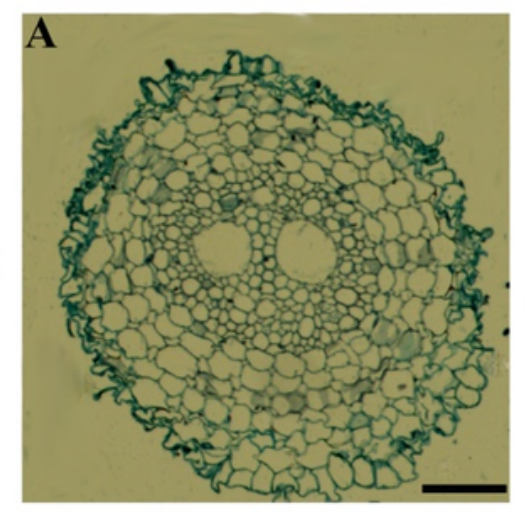

SS 212

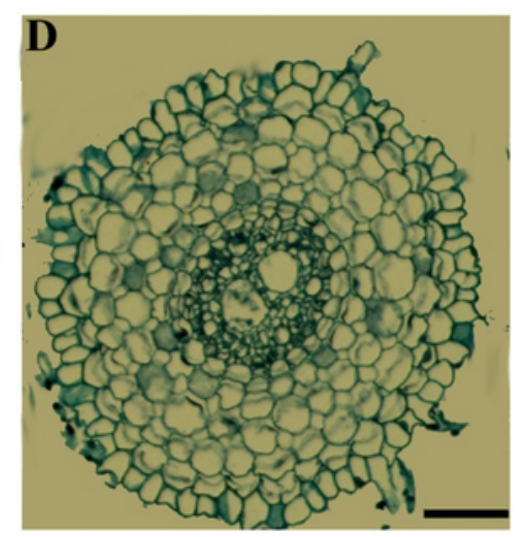

ST 47

SS 212
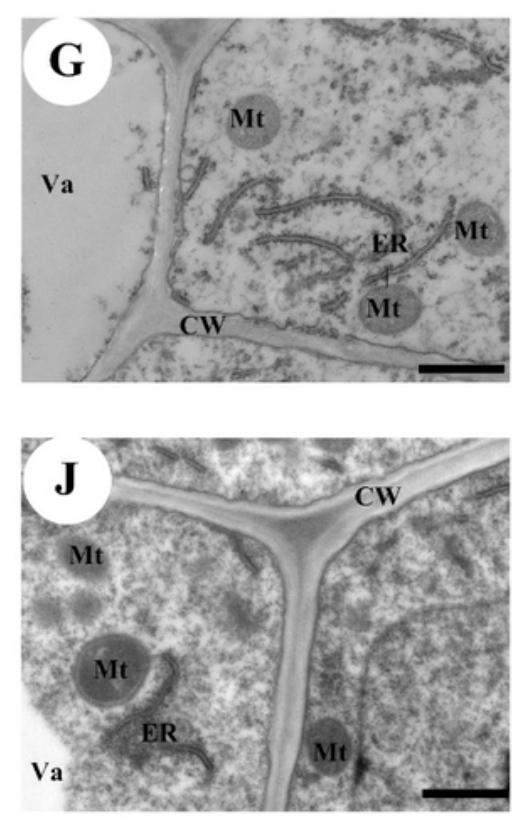

Salt stress $7 \mathrm{~d}$
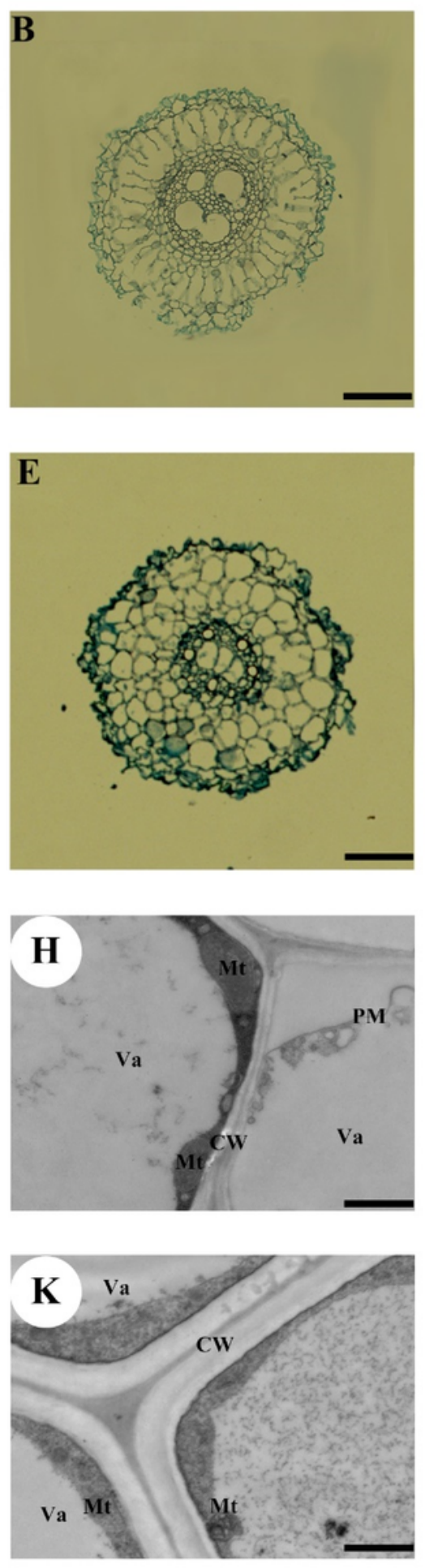

Re-watering
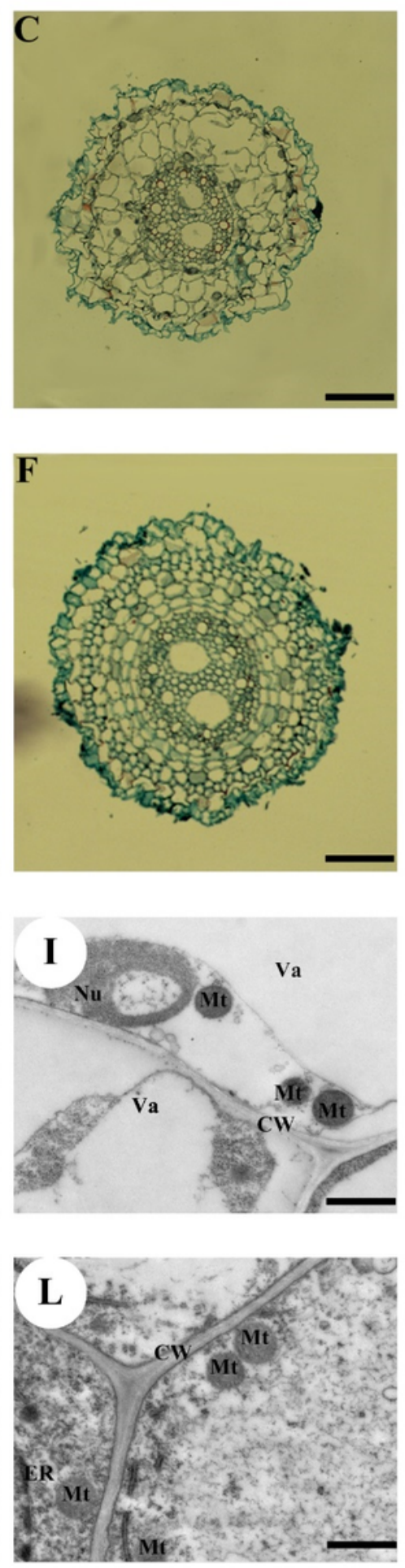

Figure 4

The root cross-section structures and subcellular structure of both proso millet cultivars under salt stress and re-watering conditions. Root cross-section structures of SS 212 (A, B, C); root cross-section structures of ST 47 (D, E, F). Control, untreated root; salt stress $7 \mathrm{~d}, 1 \% \mathrm{NaCl}$ treatment for $7 \mathrm{~d}$; Re-watering, rewatering treatment for $7 \mathrm{~d}$. Root subcellular structure of SS $212(\mathrm{G}, \mathrm{H}, \mathrm{I})$; root subcellular structure of ST $47(\mathrm{~J}, \mathrm{~K}, \mathrm{~L})$. Control, untreated root; salt stress $7 \mathrm{~d}, 1 \% \mathrm{NaCl}$ treatment for $7 \mathrm{~d}$; Re-watering, re-watering 
treatment for $7 \mathrm{~d}$. CW, cell wall; Va, vacuole; PM, plasmalemma; ER, endoplasmic reticulum; Mt, mitochondria; Nu, cell nucleus. A-F, 100 magnification, scale bars $=200 \mu \mathrm{m} ; \mathrm{G}-\mathrm{L}, 4000$ magnification, scale bars $=2 \mu \mathrm{m}$.

A

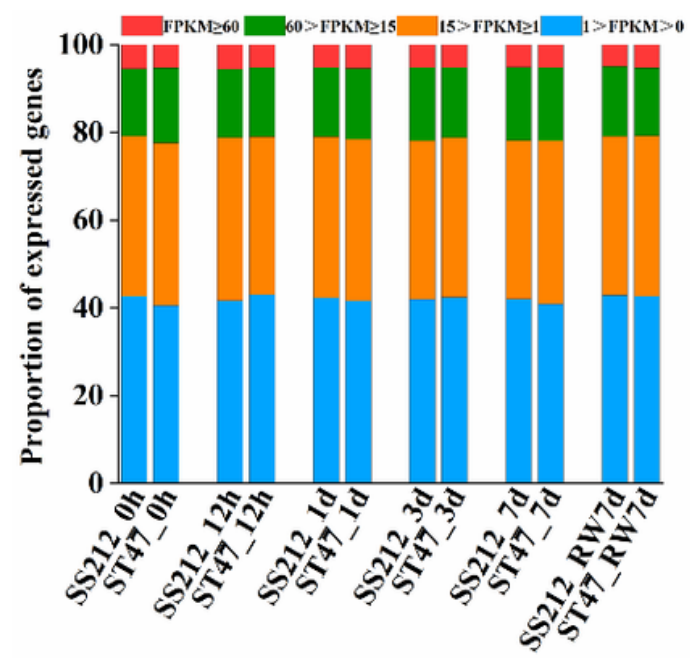

B

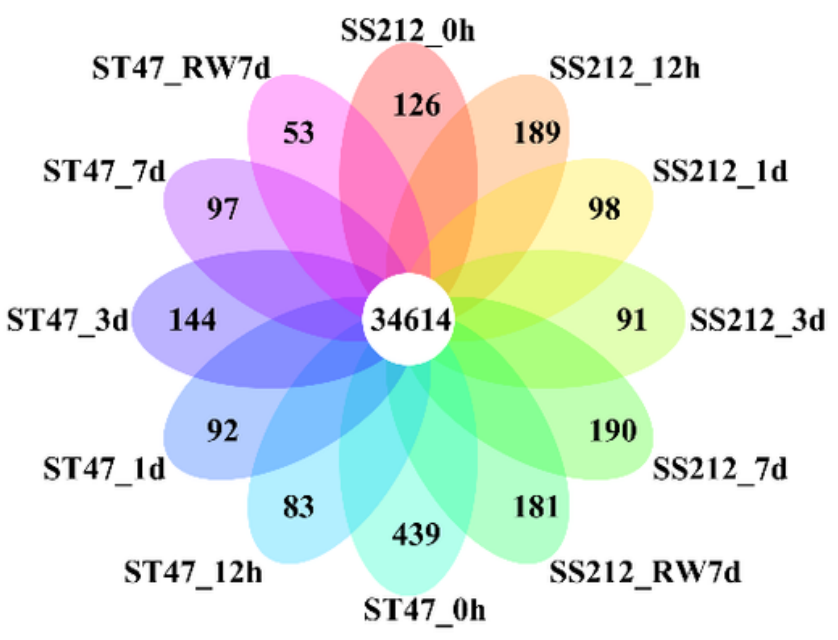

C

D
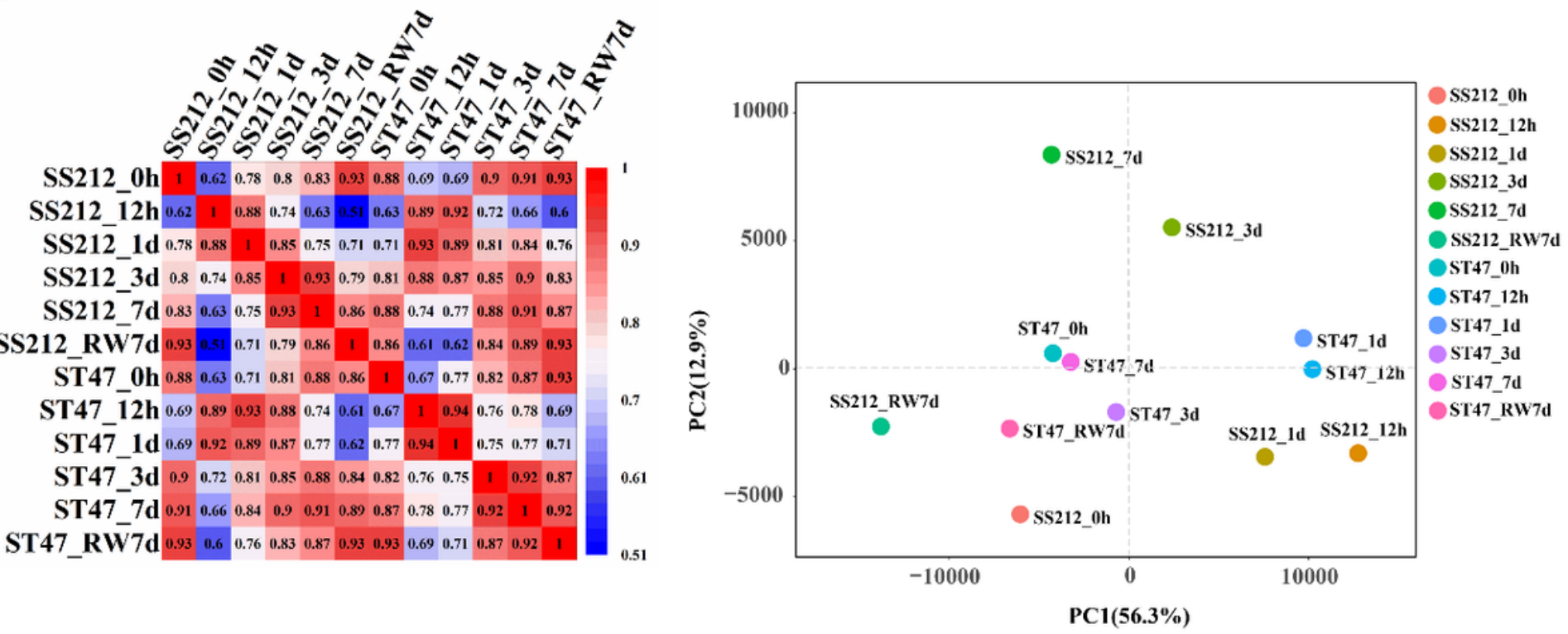

Figure 5

Gene expression profiles in 24 samples of proso millet as determined by RNA-Seq analysis. (A) Proportions of genes expressed at different levels (based on FPKM values) in 12 samples (6 time points $\times 2$ cultivars). (B) The number of transposable elements expressed in 12 samples. (C) Spearman's correlation coefficient analysis of RNA-Seq data of SS 212 and ST 47. (D) Principal component analysis (PCA) of transcriptomes of SS212 and ST 47 at $0,1 / 2,1,3$, and 7 days, and RW $7 \mathrm{~d}$. 
A

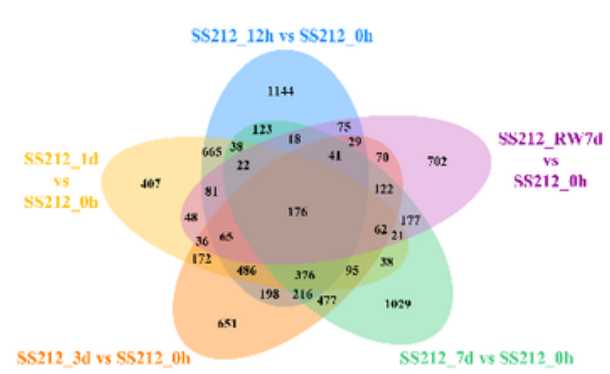

C

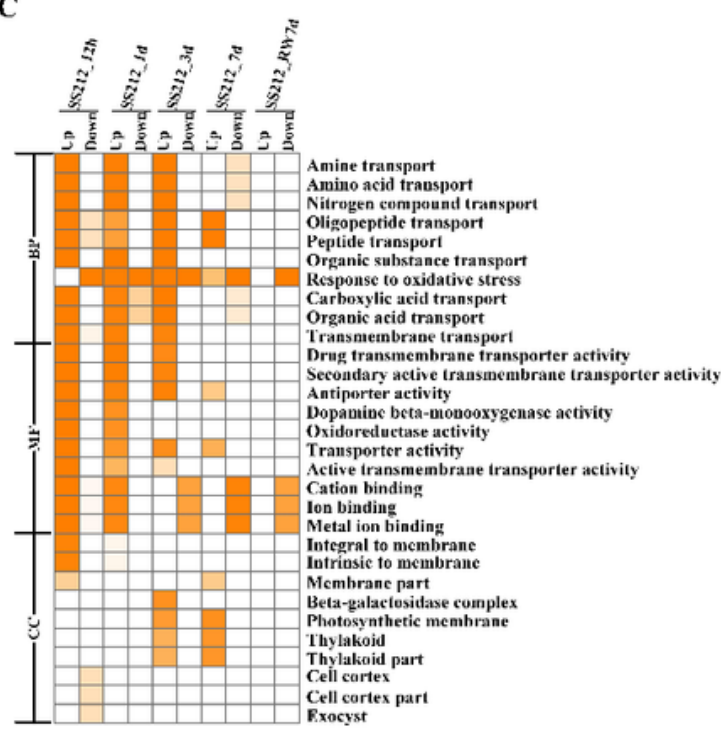

$\begin{array}{llllll}0.0 & 0.2 & 0.4 & 0.6 & 0.8 & 1.0\end{array}$

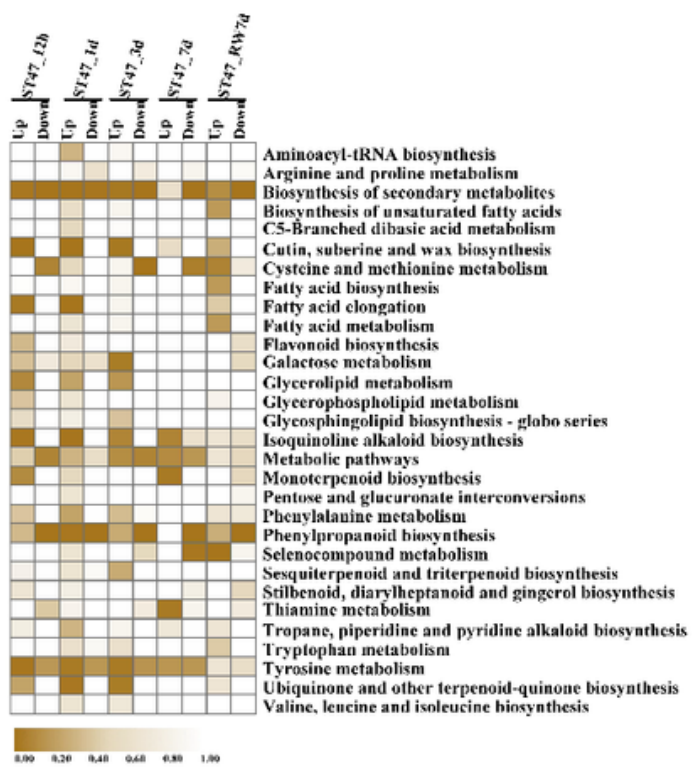

B

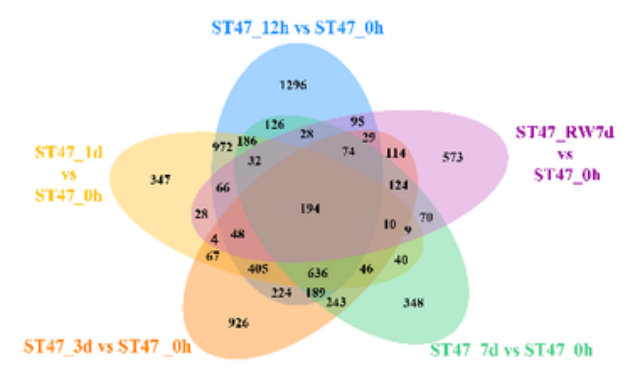

D
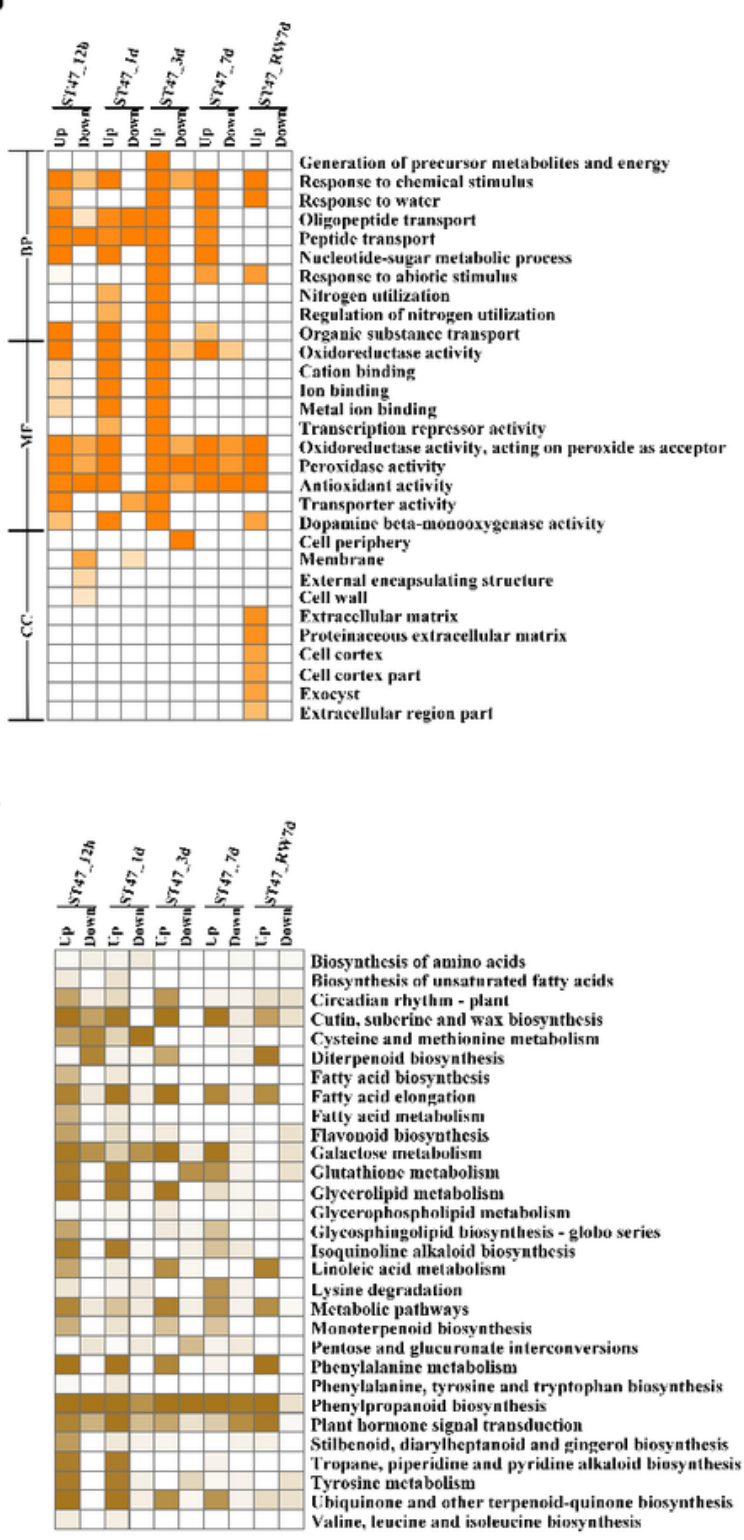

Figure 6

Differential gene expression between ST 47 and SS 212 in response to salt stress and re-watering. (A) SS 212. (B) ST 47. (C, D) Enriched GO terms (biological processes, molecular functions, and cellular components) at each treatment time point in down- and up-regulated genes in SS 212 (C) and ST 47 (D). The color scale at the bottom represents significance (corrected P-value). (E, F) KEGG pathways at each 
treatment time point in down- and up-regulated genes in SS 212 (E) and ST 47 (F). The color scale at the bottom represents significance (corrected P-value).

\section{Salt sensitive (SS 212)}

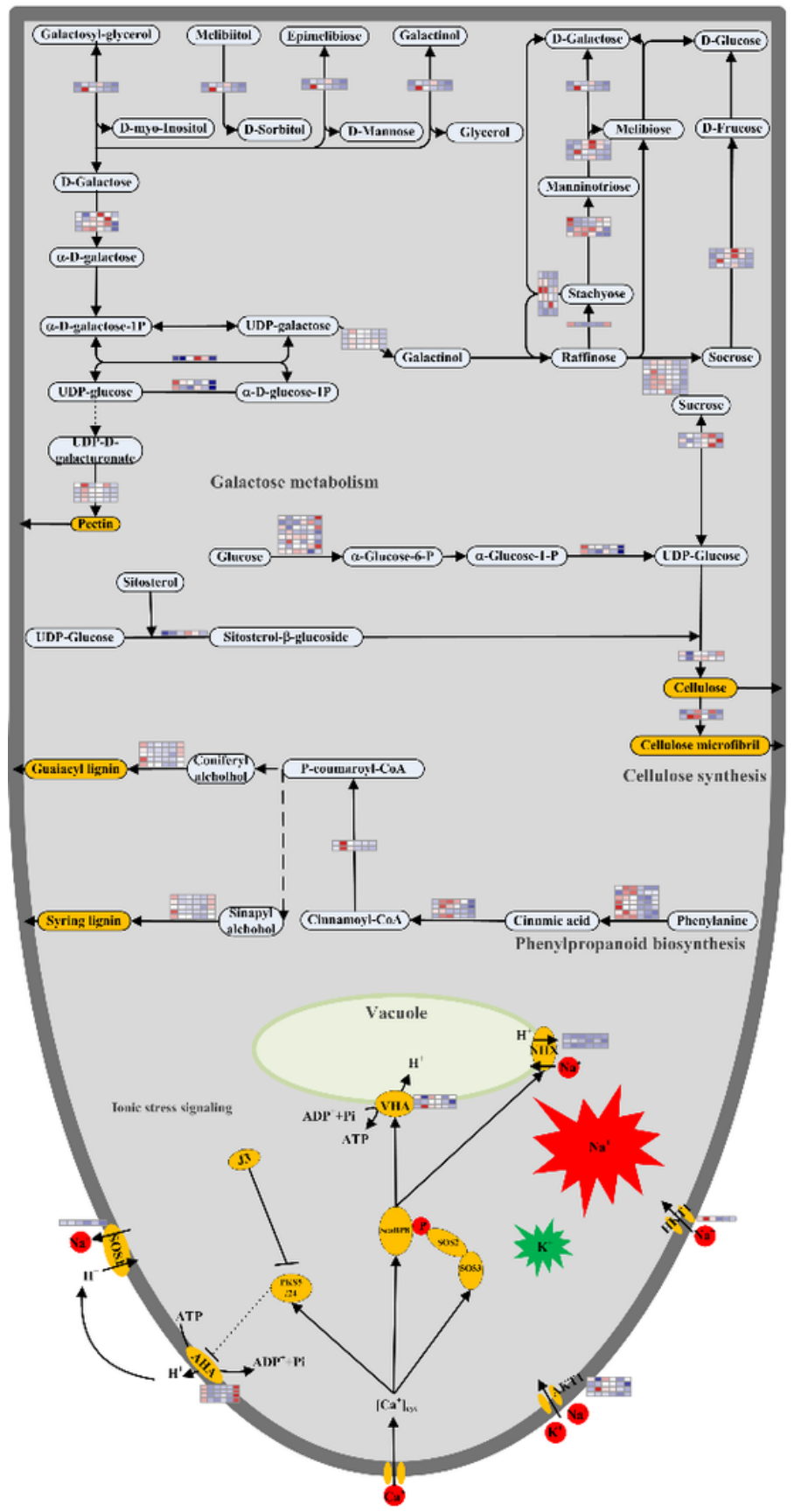

Salt tolerant (ST 47)

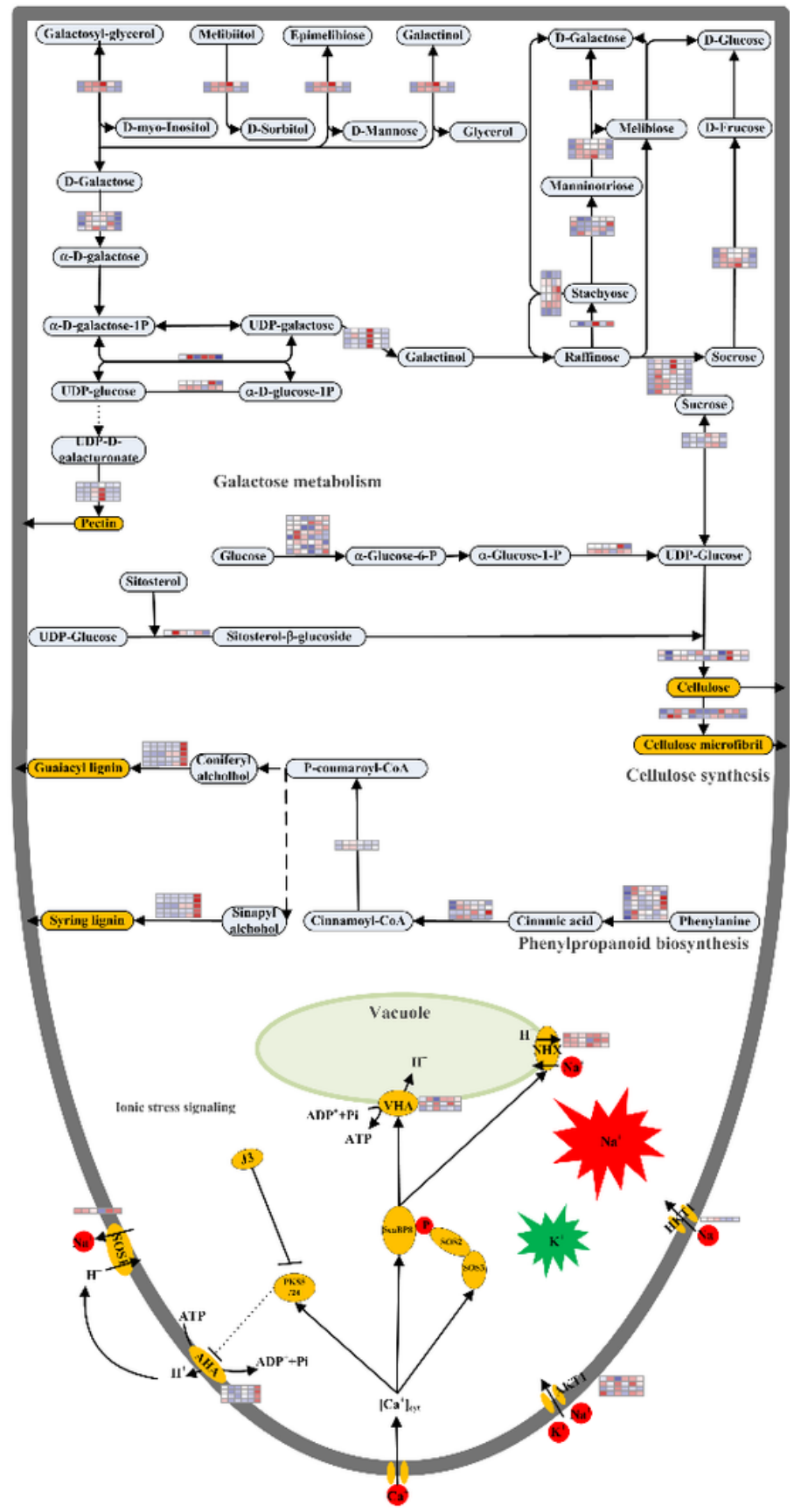

Figure 7

Transcriptional changes at each treatment time point in SS 212 and ST 47. Transcriptional changes in SS 212 and ST 47 samples at each treatment time point. Genes encoding transporter proteins, metabolites, and TFs are indicated in orange boxes, $\mathrm{Na}+\mathrm{K}+$ in red dots, and other metabolites in gray boxes. Name(s) and expression patterns of the genes encoding the corresponding enzyme(s) are indicated above or 
below the arrows. Hash arrows represent multiple enzyme reactions that were not investigated in this study.

A

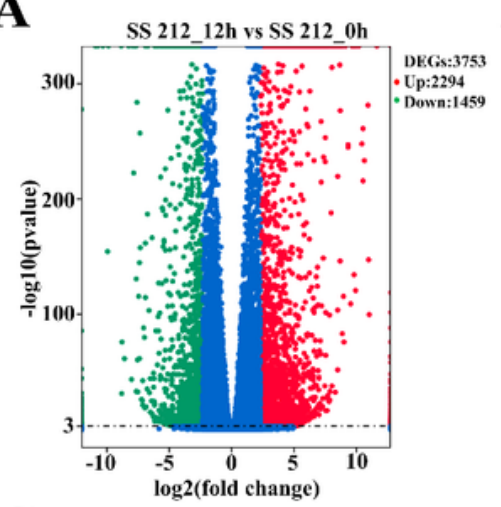

C

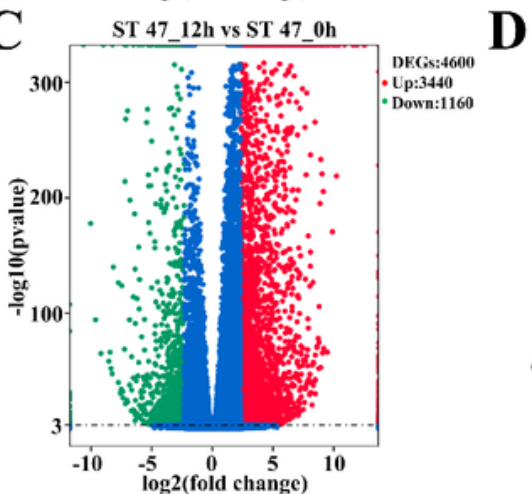

E Ss 212_RW 7d vs SS 212_0h

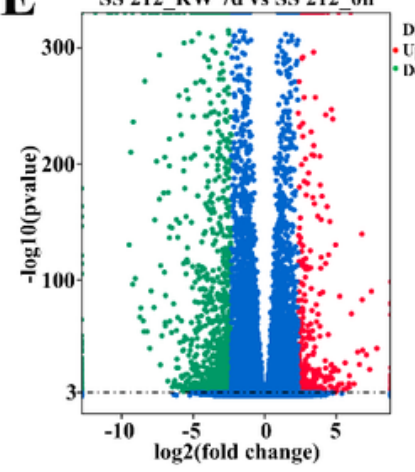

G

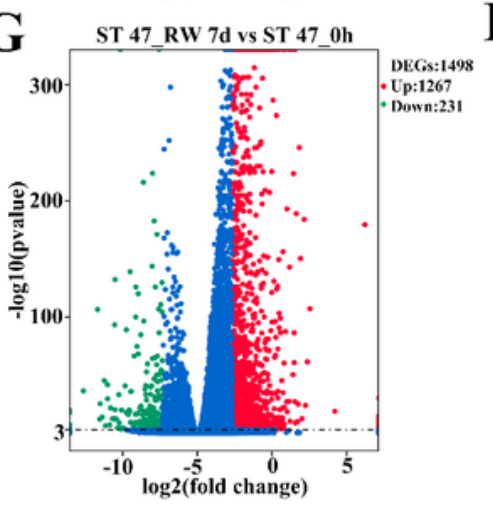

B

D

$\mathbf{F}$

$\mathbf{H}$
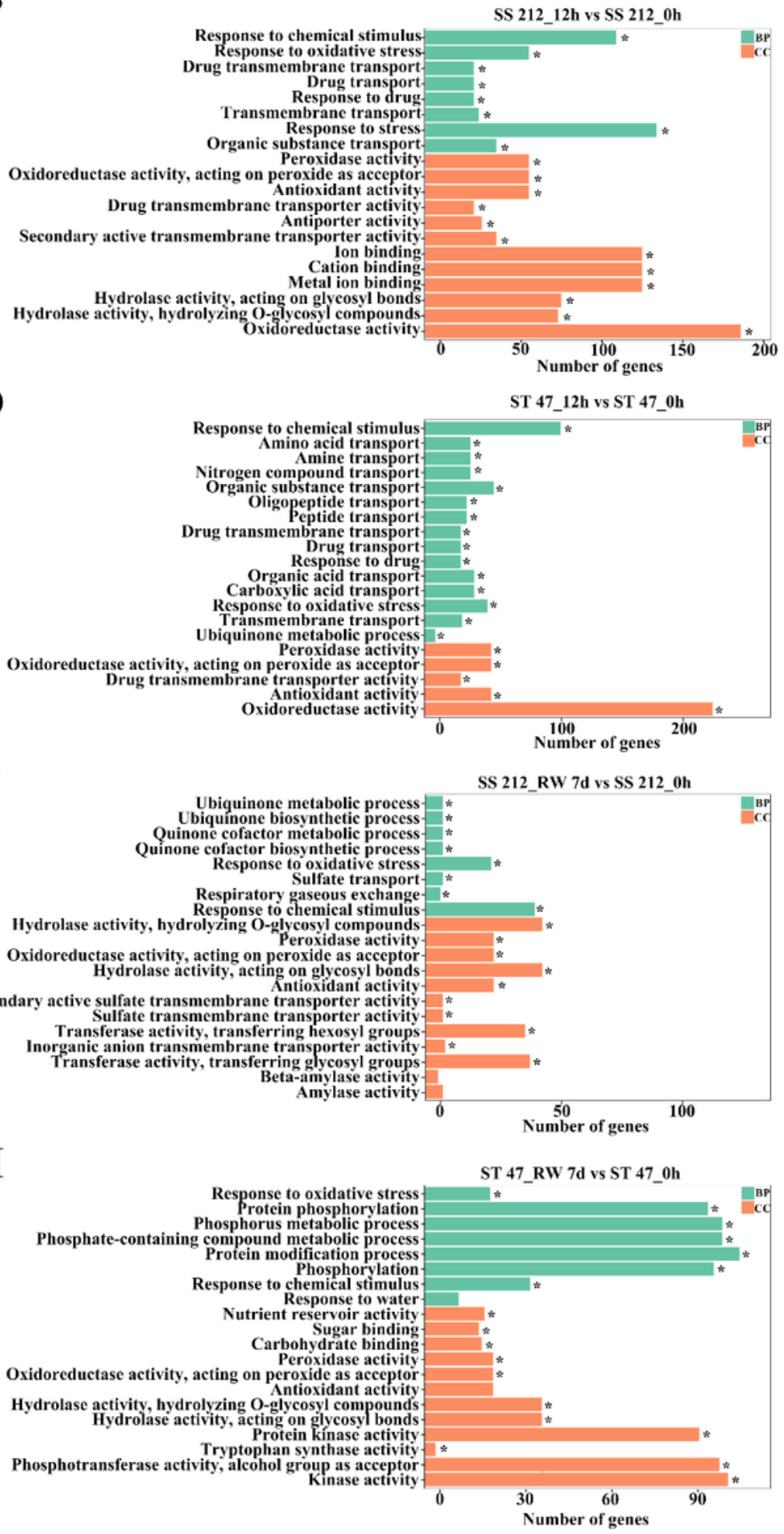

Figure 8

Differential regulation of genes between SS 212 and ST 47 in response to salt stress and re-watering. (A, C, E, G) Volcano plots of differentially expressed genes (DEGs) at $1 / 2$ day and RW $7 \mathrm{~d}$. Red and green dots represent up-regulated and down-regulated DEGs, respectively. The total number of DEGs (up- and down- 
regulated) is shown in the upper right corner. DEGs were selected based on the following criteria: $\mid \log 2$ (fold-change) $\mid \geq 1$ and false discovery rate (q-value) $\leq 0.001 ;(B, D, F, H)$. The functional categorization of up-regulated genes in both cultivars was done based on $\mathrm{GO}$ annotations.

\section{Supplementary Files}

This is a list of supplementary files associated with this preprint. Click to download.

- Supplementaltable.docx

- Supplementalfigure.docx

- SupplementaldatasetS1.xlsx

- SupplementaldatasetS2.xlsx 